\title{
Ubiquitination of tumor suppressor PML regulates prometastatic and immunosuppressive tumor microenvironment
}

\author{
Ya-Ting Wang, ${ }^{1,2}$ Jocelyn Chen,, ${ }^{1,2}$ Chou-Wei Chang, ${ }^{1}$ Jayu Jen, ${ }^{3,4}$ Tzu-Yu Huang, ${ }^{1}$ Chun-Ming Chen, ${ }^{1}$ Roger Shen, ${ }^{5}$ Suh-Yuen Liang, \\ I-Cheng Cheng, ${ }^{6}$ Shuenn-Chen Yang, ${ }^{5}$ Wu-Wei Lai, ${ }^{7}$ Kuang-Hung Cheng, ${ }^{8}$ Tao-Shih Hsieh, ${ }^{6}$ Ming-Zong Lai, ${ }^{9}$ Hung-Chi Cheng, ${ }^{3,10}$ \\ Yi-Ching Wang, ${ }^{3,4}$ and Ruey-Hwa Chen ${ }^{1,2}$ \\ IInstitute of Biological Chemistry, Academia Sinica, Taipei, Taiwan. Institute of Biochemical Sciences, College of Life Science, National Taiwan University, Taipei, Taiwan. ${ }^{3}$ Institute of Basic Medical Sciences \\ and ${ }^{4}$ Department of Pharmacology, College of Medicine, National Cheng Kung University, Tainan, Taiwan. ${ }^{5}$ Institute of Biomedical Sciences and ${ }^{\circ}$ nstitute of Cellular and Organismic Biology, Academia Sinica, \\ Taipei, Taiwan. 'Department of Surgery, National Cheng Kung University Hospital, Tainan, Taiwan. ${ }^{8}$ Graduate Institute of Biomedical Science, National Sun Yat-Sen University, Kaoshiung, Taiwan. \\ ${ }^{9}$ Institute of Molecular Biology, Academia Sinica, Taipei, Taiwan. ${ }^{10}$ Department of Biochemistry and Molecular Biology, College of Medicine, National Cheng Kung University, Tainan, Taiwan.
}

\begin{abstract}
The tumor microenvironment plays an important role in tumor growth and metastasis. However, the mechanism by which tumor cells regulate the cell and non-cell constituents of surrounding stroma remains incompletely understood. Promyelocytic leukemia ( $P M L)$ is a pleiotropic tumor suppressor, but its role in tumor microenvironment regulation is poorly characterized. PML is frequently downregulated in many cancer types, including lung cancer. Here, we identify a PML ubiquitination pathway that is mediated by WD repeat 4-containing cullin-RING ubiquitin ligase 4 (CRL4 ${ }^{\text {wDR }}$ ). Clinically, this PML degradation pathway is hyperactivated in lung cancer and correlates with poor prognosis. The WDR4/PML axis induces a set of cell-surface or secreted factors, including CD73, urokinase-type plasminogen activator receptor (uPAR), and serum amyloid A2 (SAA2), which elicit paracrine effects to stimulate migration, invasion, and metastasis in multiple lung cancer models. In xenograft and genetically engineered mouse models, the WDR4/PML axis elevates intratumoral Tregs and M2-like macrophages and reduces $\mathrm{CD} 8^{+} \mathrm{T}$ cells to promote lung tumor growth. These immunosuppressive effects were all reversed by CD73 blockade. Our study identifies WDR4 as an oncoprotein that negatively regulates PML via ubiquitination to promote lung cancer progression by fostering an immunosuppressive and prometastatic tumor microenvironment, suggesting the potential of immune-modulatory approaches for treating lung cancer with aberrant PML degradation.
\end{abstract}

\section{Introduction}

Lung cancer is one of the most devastating diseases. Despite advances in treatment, the 5-year survival rate is only slightly better than $10 \%$, and the majority of late-stage patients die within 18 months of diagnosis (1). The high mortality rate of lung cancer is mainly attributed to a failure of early diagnosis, and metastasis has frequently occurred by the time of diagnosis. Understanding the mechanism of lung cancer progression and metastasis could lead to more effective diagnosis, prognosis, and targeted therapy.

Current knowledge of tumor biology has evolved to consider the tumor an aberrant organ, in which transformed cells, along with other cell and non-cell constituents in the tumor microenvironment, conspire to facilitate tumor progression (2). Among the non-cell constituents, extracellular matrices and matrixdegrading enzymes affect many aspects of tumor behavior such as growth, survival, motility, invasion, and metastasis $(3,4)$. As to the cell constituents of stroma, immune and nonimmune cells engage in an extensive and dynamic interplay with tumor cells,

Conflict of interest: The authors have declared that no conflict of interest exists. Submitted: August 5, 2016; Accepted: May 19, 2017

Reference information: / Clin Invest. 2017;127(8):2982-2997.

https://doi.org/10.1172/JCl89957. thereby contributing to various malignant hallmarks of cancers (5). For instance, tumor-associated macrophages and Tregs play key homeostatic roles to allow tumor maintenance and growth in an immunosuppressive milieu and also diminish the effectiveness of immunotherapy (6). However, the molecular mechanism by which tumor cells regulate the functions of cell and non-cell constituents of stroma and the signals mediating the communication between tumor and stromal cells are not completely defined.

The promyelocytic leukemia (PML) protein was originally identified as a fusion partner of retinoic acid receptor $\alpha$ in acute promyelocytic leukemia (7) and is a tumor suppressor $(8,9)$. Mechanistically, PML inhibits tumor cell proliferation, migration, and invasion and promotes apoptosis and senescence (8-11). PML also regulates neoangiogenesis, cancer metabolism, and cancer stem cell maintenance (12-15). However, these multifaceted functions are largely confined to the behaviors of tumor cells. The roles of PML in regulating the tumor microenvironment and tumor immunity remain poorly characterized.

Consistent with its tumor-suppressive functions, PML protein is frequently downregulated in diverse types of human cancers including lung cancer (16). Evidence has emerged that the ubiquitin/proteasome pathway is a key mechanism for PML downregulation in tumors (17). Accordingly, aberrant PML ubiquitination and 
degradation has been observed in a number of cancer types (14, 18-22), and the ubiquitin ligases cullin 3-kelch-like family member 20 (CUL3-KLHL20) and ubiquitin protein ligase E3A (E6AP) are responsible for PML destruction in certain cancer types (18, 22). However, the ubiquitin ligase that mediates PML degradation in lung cancer remains elusive, and the molecular mechanism for PML degradation in tumors has not been completely understood.

The CUL-RING ubiquitin ligases (CRLs), including CRL1-5 and CRL7, consist of the largest family of E3 ligases. The CRL4 complex contains CUL4A or CUL4B, the ring finger protein regulator of cullin 1 (ROC1), a linker protein called damage-specific DNA-binding protein 1 (DDB1), and one of the WD repeat-containing substrate adaptors, referred to as DCAFs (DDB1- and CUL4associated factors). Most DCAFs contain a signature sequence called DWD box, which ends in a WDxR motif (23-26). In this study, we report a previously unknown PML ubiquitination pathway mediated by WD repeat 4-containing CUL-RING ubiquitin ligase $4\left(\mathrm{CRL} 4^{\mathrm{WDR}} 4\right)$ and reveal what in our view is a significant prognostic value of this pathway in lung cancer. We further show that this PML ubiquitination pathway plays a key role in lung cancer growth, progression, and metastasis by inducing a set of genes that regulate multiple constituents of the tumor microenvironment.

\section{Results}

Identification of CRL $4^{W D R} 4$ as a PML ubiquitin ligase. To identify novel PML ubiquitin ligases, we assayed PML ubiquitination in cells expressing a dominant-negative (DN) mutant of each CUL family protein. Consistent with our previous study (18), we found that CUL3-DN reduced PML ubiquitination. Interestingly, CUL4-DN elicited a similar effect (Figure 1A). PML ubiquitination was also diminished by depletion of the CRL4 component DDB1 (Supplemental Figure 1A; supplemental material available online with this article; https://doi.org/10.1172/JCI89957DS1). To search for the substrate adaptor that mediates CRL4-dependent PML ubiquitination, we individually depleted 13 reported or predicted DCAFs. This screen revealed a marked decrease in PML ubiquitination by WDR4 knockdown (Supplemental Figure 1B). We observed similar effects in another cell line and by transfecting cells with a WDR4 siRNA (Figure 1B). Reciprocally, WDR4 overexpression enhanced the ubiquitination of PML-I and PML-IV (Figure 1C). WDR4 is a WD-repeat protein that possesses a signature sequence of DCAFs (Supplemental Figure 1C). Consistent with the DCAF characteristics, WDR4 carrying a mutation in the WDxR motif (R219A) showed a reduced ability for DDB1 binding and CUL4/ DDB1-mediated autoubiquitination (Figure 1D and Supplemental Figure 1D). This mutant was also less effective at promoting PML ubiquitination than was the WT protein (Supplemental Figure 1E). We further showed that WDR4 was capable of binding exogenous and endogenous PML (Figure 1E and Supplemental Figure $1 \mathrm{~F}$ ) and that WDR4 depletion compromised PML binding to endogenous DDB1, CUL4A, and CUL4B (Figure 1F). These findings collectively support the notion that WDR4 functions as a CRL4 substrate adaptor that recruits PML to the CUL4-DDB1 complex. Next, we sought to determine whether PML is a direct substrate of CRL $4^{\mathrm{WDR} 4}$. Glutathione-S-transferase (GST) pull-down analysis demonstrated a direct interaction between purified PML and WDR4 (Figure $1 G)$. Furthermore, an in vitro ubiquitination assay showed that
PML was efficiently ubiquitinated in the presence of a CUL4A or CUL4B complex containing ROC1 (a CUL4 complex subunit), DDB1, and WDR4 (Figure 1H). Thus, our study identifies CRL4 $4^{\text {WDR4 }}$ as a ubiquitin ligase for PML.

To determine the region in WDR4 that is critical for PML binding, we performed a structural modeling of WDR4 using the tertiary structure of TRM82 (27), the yeast ortholog of WDR4, as a template. The WD-repeat domain of WDR4 adopts a 7-blade $\beta$-propeller structure, in which the WDxR motif is localized to the bottom surface of the $\beta$-propeller (Supplemental Figure 2A). Because of the possible steric hindrance between DDB1 and substrate binding and the frequent utilization of the top surface for peptide binding (28), we deleted a few residues in each of the 14 linkers on the top surface (Supplemental Figure 2B). An in vitro binding assay revealed that most of these mutants displayed a reduction in PML-I binding, with dTL1, dTL3, and dTL11 showing the most dramatic effect (Supplemental Figure 2C). Accordingly, dTL1 showed a marked defect in promoting PML ubiquitination (Supplemental Figure 2D), further supporting the idea of a direct role for CRL4 $4^{\mathrm{WDR} 4}$ in PML ubiquitination.

WDR4 promotes $P M L$ proteasomal degradation. Next, we investigated the consequence of PML ubiquitination by CRL $4^{\mathrm{WDR} 4}$. WDR4 overexpression in multiple cell lines reduced PML abundance, whereas the WDR4 R219A and dTL1 mutants did not elicit a significant effect (Figure 2, A and B, and Supplemental Figure 2E). Conversely, WDR4 knockdown in multiple cell types, including the lung cancer cell lines A549 and H1299 and the patient-derived primary lung cancer cells CL152 and CL141, markedly increased PML levels (Figure 2C and Supplemental Figure 5A). WDR4 knockdown in normal human bronchus epithelial BEAS-2B cells led to a modest increase in PML abundance, presumably because of its low WDR4 and high PML levels (Figure 2C). A cycloheximide (CHX) chase experiment showed that WDR4 overexpression increased PML-I turnover, whereas WDR4 knockdown stabilized endogenous PML levels (Figure 2, D and E). Furthermore, proteasome inhibition induced a greater PML stabilization in cells carrying WT WDR4 than did cells with a WDR4 R219A mutant (Figure 2F). These findings indicate that WDR4 potentiates PML proteasomal degradation.

The WDR4/PML axis is hyperactivated in lung cancer and correlates with a poor prognosis. Having identified a WDR4-dependent PML degradation pathway, we next explored its clinical significance in human cancers. Immunohistochemical analysis of a tissue microarray of multiple types of cancers derived from patients revealed possible high expression levels of WDR4 in lung cancer (Supplemental Figure 3A). Furthermore, by analyzing The Cancer Genome Atlas (TCGA) data set for patients with lung adenocarcinoma (including 512 primary tumor tissues and 59 adjacent normal tissues), we found upregulated WDR4 mRNA in all stages of tumor tissues compared with mRNA levels in normal tissues (Figure 3A). In addition, higher WDR4 mRNA levels correlated with poor patient survival (Figure 3B). We obtained similar findings by analyzing microarray data sets of lung cancer cohorts retrieved from the NCBI's Gene Expression Omnibus (GEO) database (Supplemental Figure 3, B and C). Since PML protein is prevalently downregulated in lung cancer (16), we focused on this cancer type. We assessed the protein expression of WDR4 and PML in a cohort of 120 lung cancer patients. Immunohistochemical analy- 
A

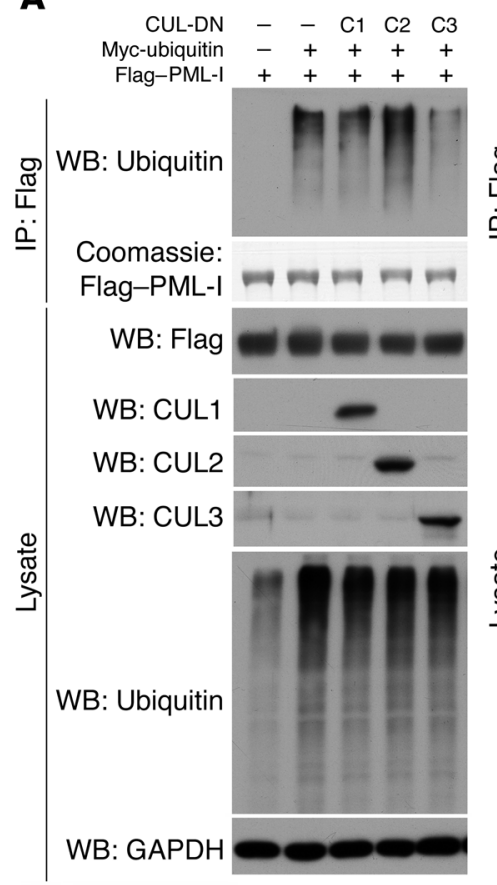

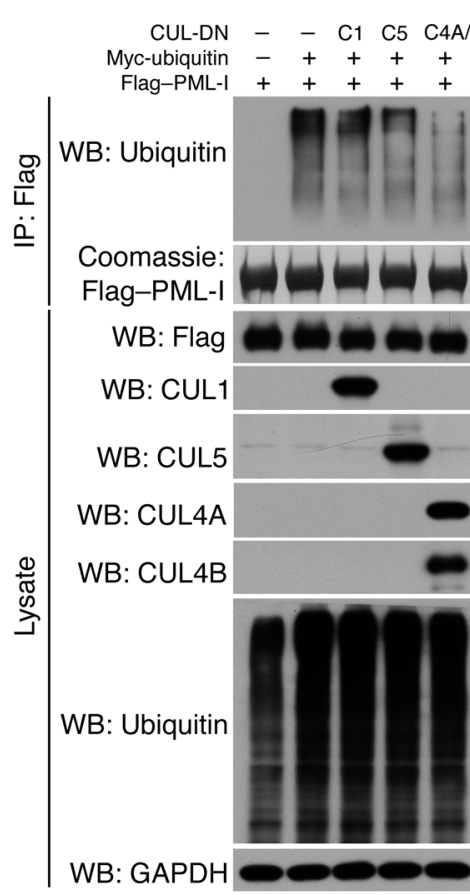

B
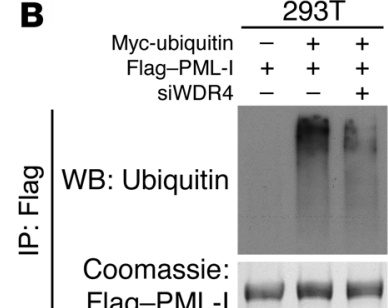

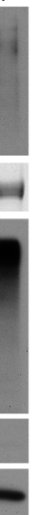

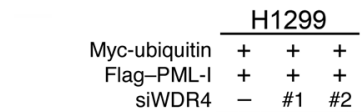

WB: Ubiquitin

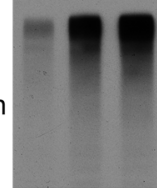

WB: WDR4

WB: GAPDH - -
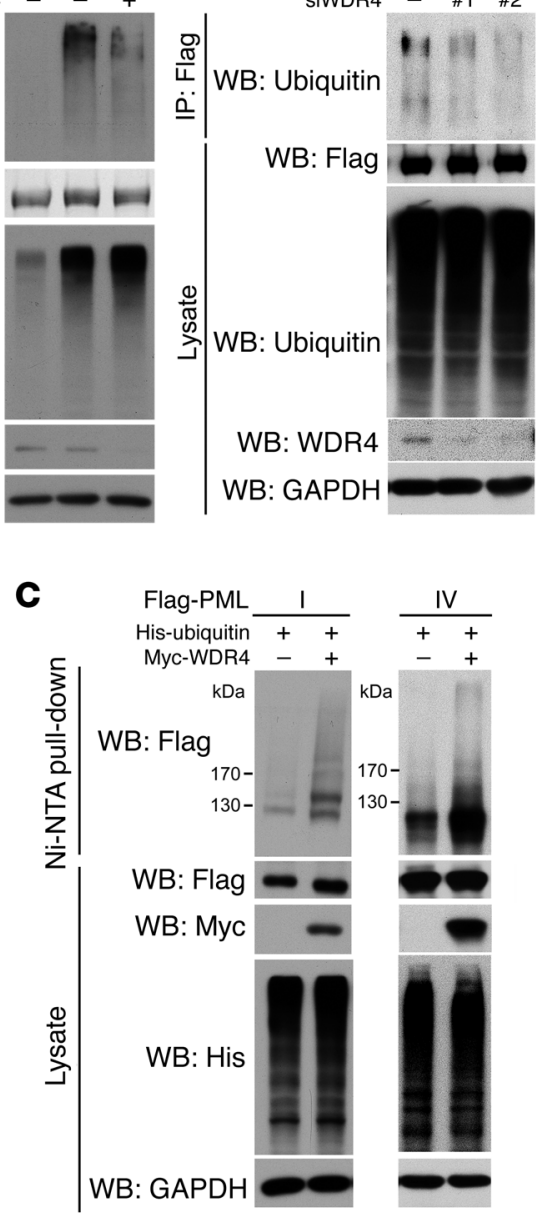

D

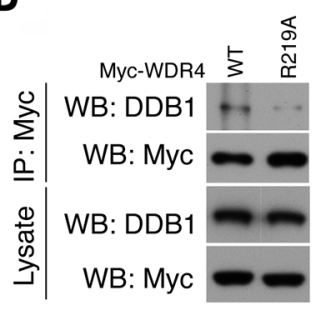

$\mathbf{F}$

F Flag-PML-I +++

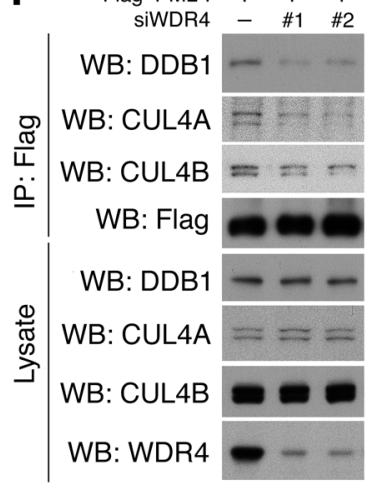

E

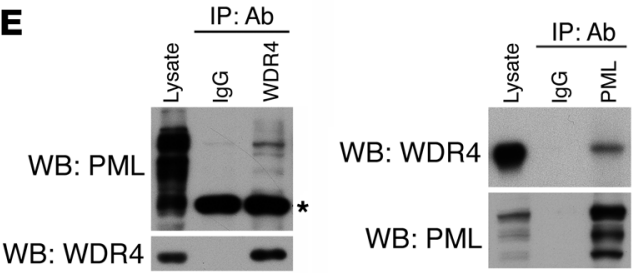

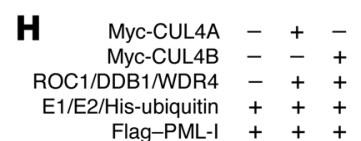

Flag-PML-I

WB: Flag

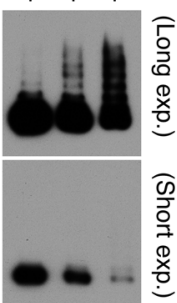

\section{Input E3 ligase complex}

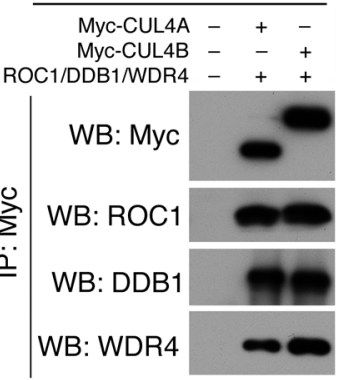

Figure 1. PML is a substrate of CRL4 ${ }^{\text {woR4 }}$. (A and B) Immunoprecipitation analysis for PML ubiquitination in $293 \mathrm{~T}$ cells transfected with the indicated constructs and/or siRNA or in $\mathrm{H} 1299$ lung cancer cells stably expressing WDR4 shRNA and transfected with the indicated constructs. (C) Ni-nitrilotriacetic acid (Ni-NTA) pull-down analysis for PML-I and PML-IV ubiquitination in 293T cells transfected with the indicated constructs. (D) Immunoprecipitation analysis of the interaction between WDR4 or its mutant (R219A) and endogenous DDB1 in 293T cells. (E) Reciprocal immunoprecipitation analysis of the interaction between endogenous WDR4 and endogenous PML in $\mathrm{H} 1299$ lung cancer cells. The asterisk marks a nonspecific band. (F) Immunoprecipitation analysis of the interaction between PML-I and endogenous DDB1, CUL4A, and CUL4B in 293T cells transfected with the PML-I construct and/or WDR4 SiRNA. (G) GST pull-down analysis of the in vitro interaction between GST-WDR4 and baculovirally purified PML-I. (H) In vitro ubiquitination assay for baculovirally purified PML-I incubated with the WDR4-based CUL4A or CUL4B ubiquitin ligase. The equal input of the CUL4A and CUL4B complexes is shown on the right. exp., exposure; WB, Western blot. 
A

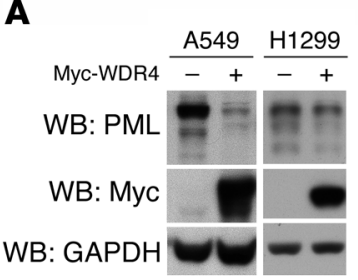

B

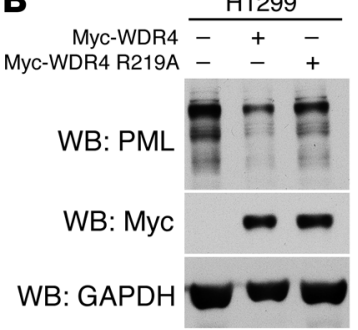

C

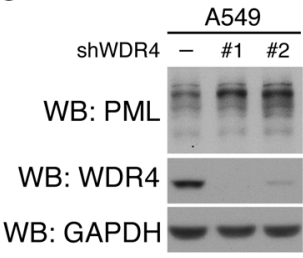

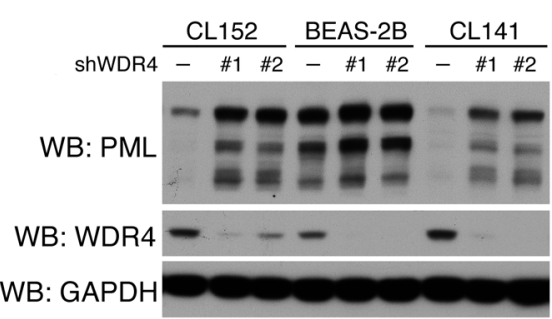

$\mathbf{F}$
Flag-PML-I ++++++++

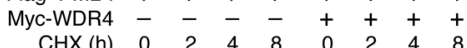
$\begin{array}{lllll}\mathrm{CHX}(\mathrm{h}) & 0 & 2 & 4 & 8\end{array}$

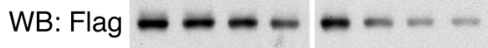
$\begin{array}{llllllll}1.00 & 0.94 & 0.82 & 0.62 & 1.00 & 0.55 & 0.32 & 0.26\end{array}$

WB: Myc

WB: GAPDH
E

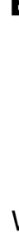

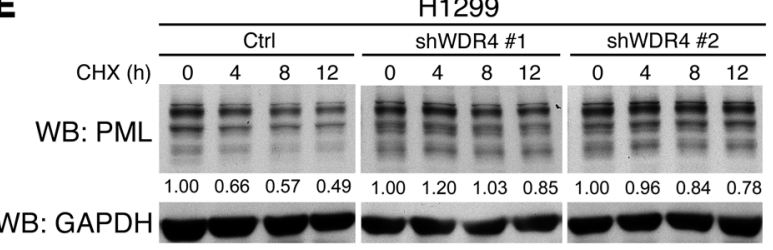

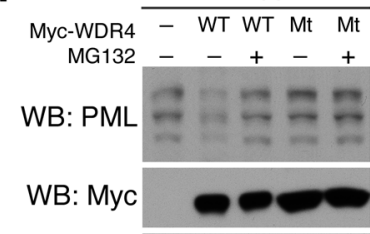

WB: GAPDH

Figure 2. WDR4 promotes PML proteasomal degradation. (A-C) Western blot analysis of endogenous PML in the indicated cell lines or in patient-derived primary lung cancer cells (CL152 and CL141) transfected with WDR4 or its mutant or stably expressing WDR4 shRNAs. (D and E) Western blot analysis of PML-I stability in CL1-0 lung cancer cells transfected with PML-I and WDR4 and endogenous PML stability in H1299 cells stably expressing WDR4 shRNA. The relative amounts of PML are indicated. The knockdown efficiencies of WDR4 shRNAs are shown in Supplemental Figure 5A. (F) Western blot analysis of endogenous PML in H460 cells transfected with WDR4 or the WDR4 mutant R219A (Mt) and treated with MG132 for 16 hours.

sis revealed nuclear staining for both WDR4 and PML (Figure 3C, insets). Compared with the adjacent normal lung tissues, tumor tissues showed significant upregulation of WDR4 and downregulation of PML (Figure 3C). Furthermore, high WDR4 expression levels correlated with low PML expression levels (Figure 3D). These data support a frequent hyperactivation of the WDR4/PML axis in lung cancer. Importantly, high expression of WDR4, low expression of PML, or a combination of high WDR4 and low PML expression all correlated with poor disease-free survival (Figure $3, E-G)$. Our findings indicate the prognostic value of WDR4 and PML expression in lung cancer and suggest a role for the WDR4/ PML axis in lung malignancy.

The WDR4/PML axis induces a set of tumor-promoting factors. To explore the functional impact of the WDR4/PML axis on lung malignancy, we performed cDNA microarray analysis to identify genes whose expression was coregulated by WDR4 overexpression and PML knockdown in the A549 lung cancer cell line. Our data indicated that the transcripts of 1,716 genes and 328 genes were altered by PML knockdown and WDR4 overexpression, respectively. The weaker effect elicited by WDR4 overexpression versus PML knockdown may have been the result of a milder reduction in PML expression by WDR4 overexpression than by PML siRNA (Supplemental Figure 4A). Nevertheless, 36 transcripts were coincidently regulated by WDR4 overexpression and PML knockdown (Figure 4A). Gene ontology (GO) analysis of these genes revealed that wound healing, cell motion, and locomotory behavior are among the significantly overrepresented biological processes (Figure 4B), suggesting an effect of the WDR4/ PML axis on cell migration. We validated several tumor-promoting genes in the upregulated group and tumor-suppressive genes in the downregulated group by reverse transcription quantitative PCR (RT-qPCR) and Western blot analyses. These analyses demonstrated that $\mathrm{CD} 73$, urokinase-type plasminogen activator receptor (UPAR), and serum amyloid A2 (SAA2) were most prominently and consistently upregulated by WDR4 overexpression and PML knockdown in A549 and H460 lung cancer cells (Figure 4, C and D, Supplemental Figure 4B and data not shown). WDR4 R219A and WDR4 dTL1, which could not cause PML downregulation, did not significantly elevate the expression of these 3 proteins (Supplemental Figure 4C). In the reciprocal experiment, WDR4 knockdown in A549 cells and patient-derived primary lung cancer cells decreased the expression levels of CD73, UPAR, and SAA2, which were all reversed by PML knockdown (Figure $4 \mathrm{E}$ and Supplemental Figure 4D). These findings identify 3 downstream effectors for the WDR4/PML axis.

CD73, also known as NT5E, is a membrane-bound nucleotidase that catalyzes the conversion of extracellular AMP to adenosine, which binds to adenosine receptors on the surface of immune cells to elicit profound immunosuppressive effects (29). Additionally, CD73 enhances tumor migration, invasion, and metastasis at least partly through an immune-independent mechanism (30). uPAR participates in the plasminogen activation system to promote extracellular matrix (ECM) proteolysis and functions as a coreceptor of integrin to enhance ECM signaling (31). Serum amyloid A (SAA) family proteins, including SAA2, promote tumor migration, invasion, and metastasis through multiple mechanisms, including the promotion of ECM proteolysis via plasminogen activation (32). Thus, these 3 proteins can each contribute to the establishment of a prometastatic tumor microenvironment to enhance the metastasis-related traits of tumor cells. In line with their tumor-promoting functions, our analysis of TCGA data set revealed $C D 73$ and SAA2 upregulation in patients with certain stages of lung adenocarcinoma (Supplemental Figure 4E). Furthermore, our analysis of both TCGA lung adenocarcinoma and GEO lung cancer data sets revealed that high expression levels of either CD73 or UPAR correlated with a poor prognosis (Sup- 
A

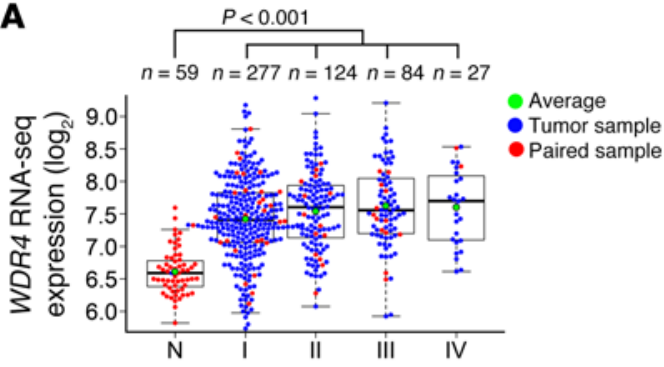

B

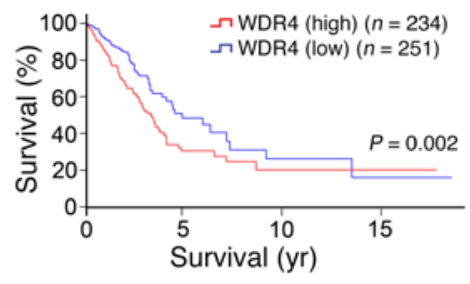

E

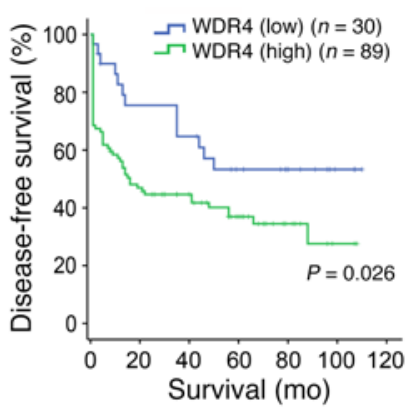

C Adjacent normal
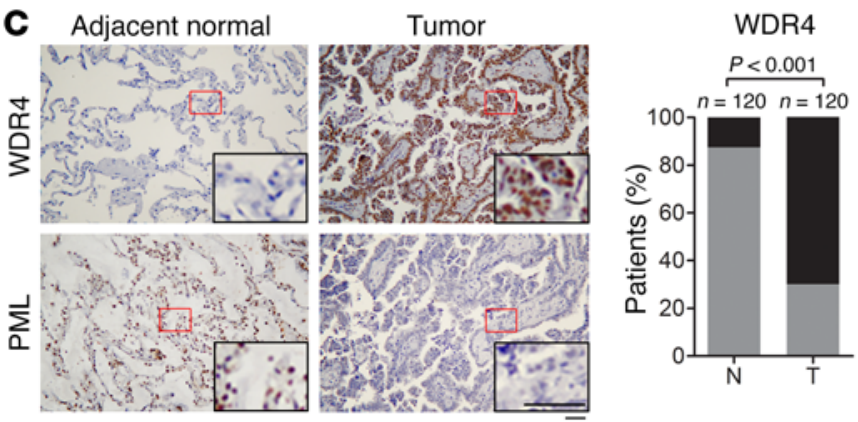

PML

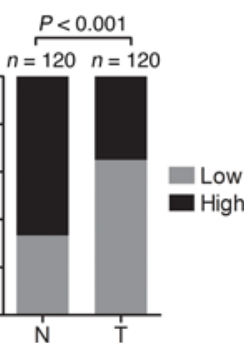

D

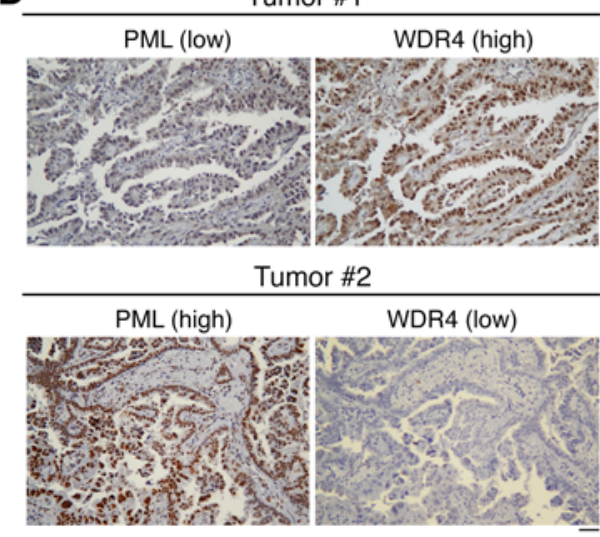

Tumor \#1

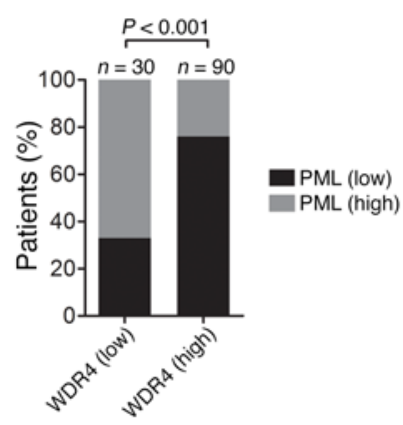

$\mathbf{F}$

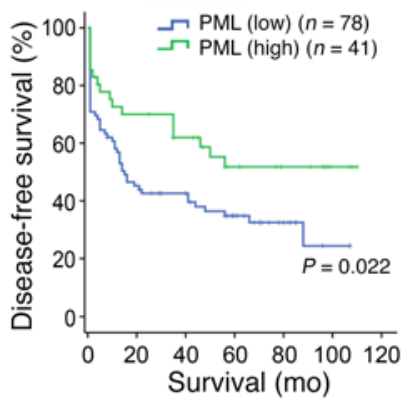

G

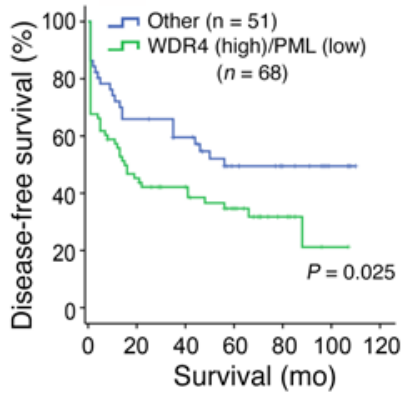

Figure 3. WDR4 upregulation correlates with PML downregulation and poor prognosis in lung cancer. (A) Beeswarm boxplot presentation of the expression profiles for WDR4 mRNA in lung adenocarcinoma tissues and adjacent normal tissues derived from TCGA data set. N, normal. (B) Kaplan-Meier analysis of lung adenocarcinoma patients' survival, with the corresponding expression profiles derived from TCGA data set. High and low expression was defined using an optimized cut point. (C) Representative immunohistochemical data for WDR4 and PML expression in lung tumor tissue and its adjacent normal tissue. Scale bars: $20 \mu \mathrm{m}$. The boxed areas are enlarged to show nuclear staining for both PML and WDR4. Bar graph shows a summary of the WDR4 and PML expression profiles for 120 lung tumor tissues $(T)$ and their adjacent normal tissues $(N)$. (D) Representative immunohistochemical images for 2 lung tumor tissues showing an inverse relation of WDR4 and PML expression. The inverse correlation of WDR4 expression with PML expression in 120 lung tumor specimens is shown on the right. Scale bar: $20 \mu \mathrm{m}$. (E-G) Kaplan-Meier analysis of lung cancer patients' survival with the corresponding expression profiles. One patient without survival data was excluded. $P$ values were determined by 1-way ANOVA with Tukey's post test (A), Fisher's exact test (C and $\mathbf{D})$, and log-rank test (B and $\mathbf{E}-\mathbf{G})$.

plemental Figure 4, F and G). Notably, both CD73 and uPAR are downstream targets of HIF-1 $(33,34)$, and the level of SAA2 was also induced under hypoxic conditions in an HIF-1 $\alpha$-dependent manner (Supplemental Figure $4 \mathrm{H}$ ). Since PML is known to suppress HIF-1 $\alpha$ translation (12), we tested whether the WDR4/ PML axis regulates CD73, uPAR, and SAA2 through HIF-1. Importantly, we found that depletion of HIF-1 $\alpha$ attenuated the induction of CD73, uPAR, and SAA2 by WDR4 overexpression and PML knockdown in both normoxic and hypoxic conditions (Figure 4, F and G). These data indicate that WDR4-mediated PML degradation upregulates HIF-1 to induce these 3 effectors.
Since UPAR and SAA2 can each induce the activation of MMPs through plasminogen activation $(32,35,36)$, we examined whether the WDR4/PML axis could regulate the activity of certain MMPs in conditioned medium (CM). Remarkably, MMP2 and MMP9 activity was consistently upregulated by WDR4 overexpression or PML knockdown (Figure 4, H and I). Thus, our study demonstrated that the WDR4/PML axis induces the expression or activation of a set of tumor-promoting factors including CD73, uPAR, SAA2, MMP2, and MMP9, each of which is known to enhance metastasis-related traits such as migration and invasion. 
A

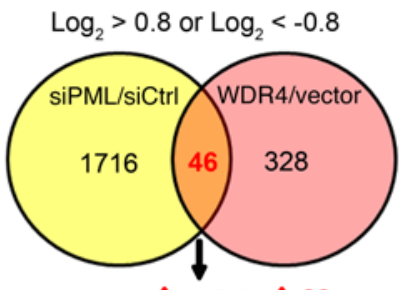

SiPML $\uparrow ; W D R 4 \uparrow: 22$

siPML $\downarrow$;WDR4 $\downarrow: 14$

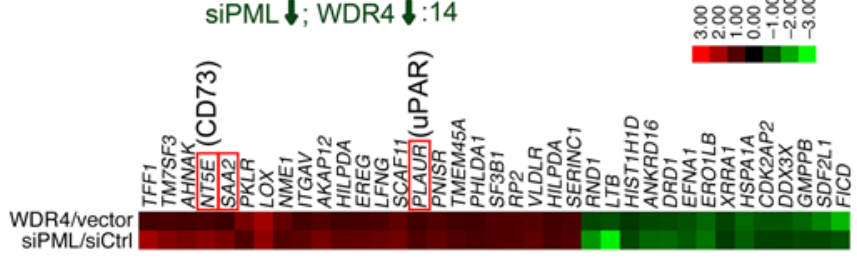

B

GO term: biological process

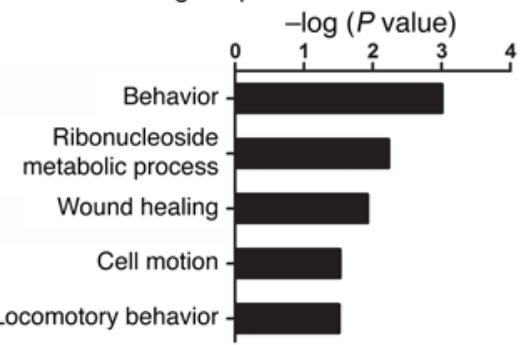

Locomotory behavio
C

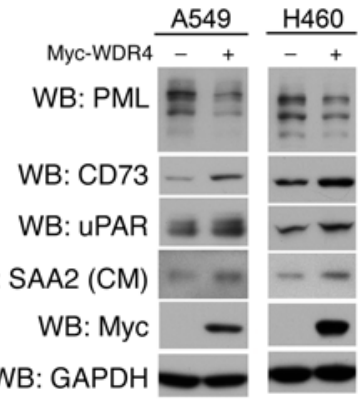

D

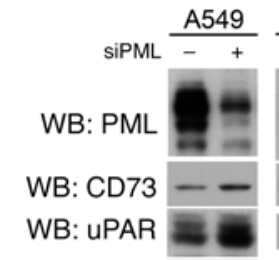

WB: SAA2 (CM)

WB: GAPDH
E

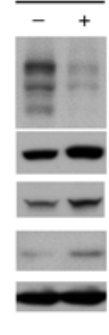

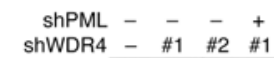

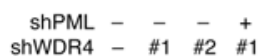

WB: PML

WB: $C D 73$

WB: UPAR

WB: SAA2 (CM)

WB: WDR4

WB: GAPDH

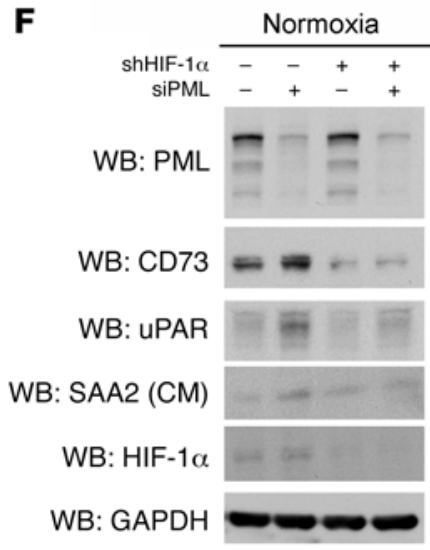

H

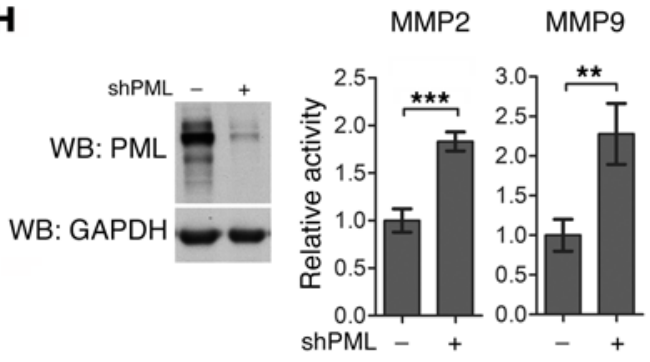

G

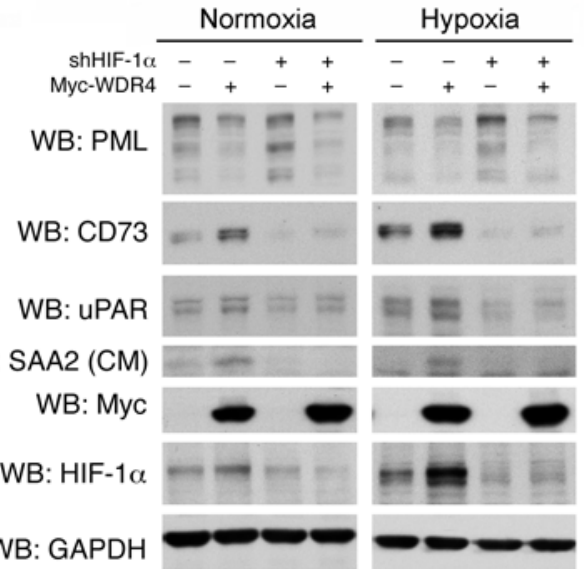

I
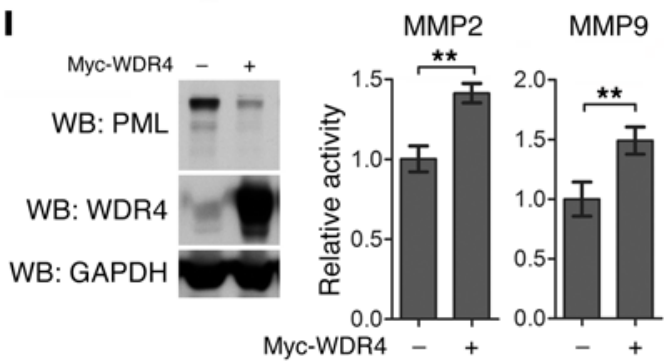

Figure 4. The WDR4/PML axis induces several tumor-promoting genes. (A) Summary of the microarray analysis of genes regulated by WDR4 overexpression or PML knockdown in A549 cells. Heatmap shows the 36 genes that are regulated coincidently by WDR4 overexpression and PML knockdown. Ctrl, control. (B) Representative $G 0$ terms specifically enriched in genes coregulated by WDR4 overexpression and PML knockdown. (C and D) Western blot analysis of the indicated proteins in A549 and H460 lung cancer cells transfected with the WDR4 construct or PML siRNA. CM was used to detect secreted SAA2. (E) Western blot analysis of the indicated proteins in A549 cells stably expressing the indicated shRNAs. (F and G) Western blot analysis of the indicated proteins in $\mathrm{H} 1299$ cells stably expressing control or HIF-1 $\alpha$ shRNA, transfected with PML siRNA or the WDR4 construct, and cultured in hypoxic or normoxic conditions for 24 hours. (H and I) MMP2 and MMP9 activity in CM derived from A549 cells stably expressing PML shRNA or the WDR4 construct. Data represent the mean \pm SD; $n=3$ per group. ${ }^{* *} P<0.01$ and ${ }^{* *} P<0.001$, by 2 -tailed Student's $t$ test. Western blots show WDR4 and PML expression levels. 


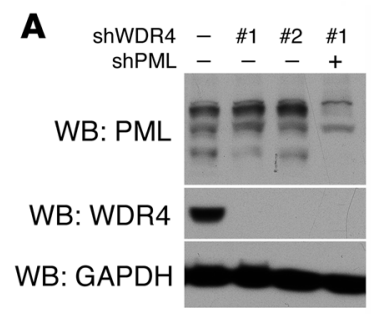

E

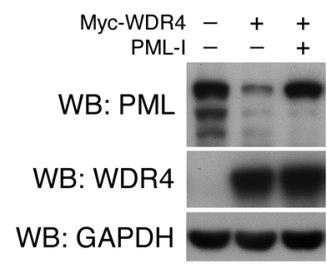

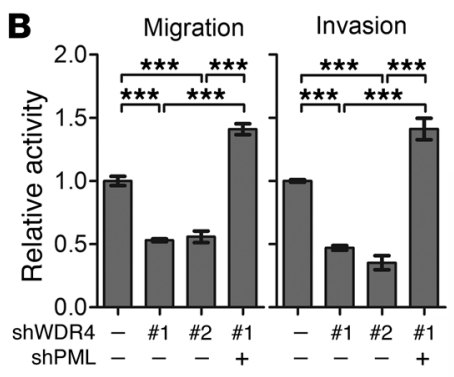

F Migration Invasion

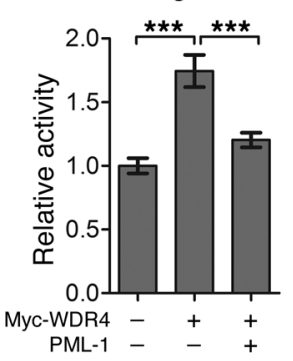

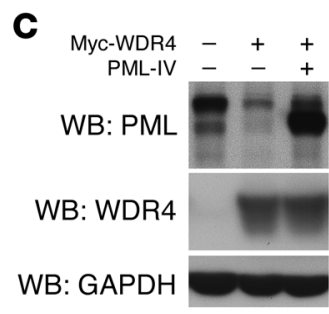
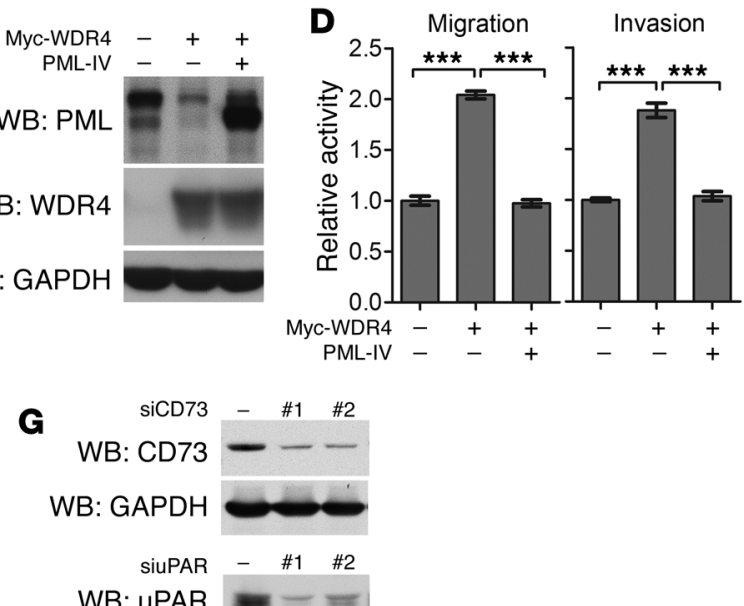

WB: GAPDH

SISAA2 - \#1 \#2

WB: SAA2

WB: GAPDH
H

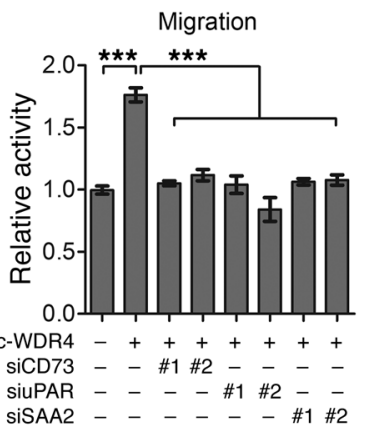

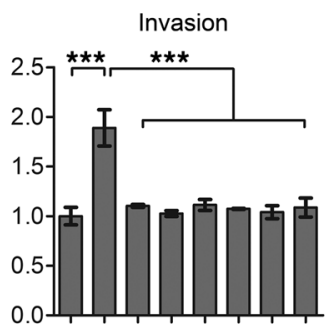

$-++++++$

- - \#1\#2 - - -

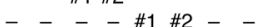

$\begin{array}{llll}- & - & -\end{array}$ \#2 $-\overline{-}$

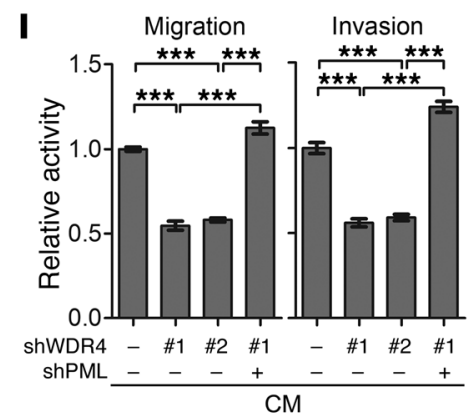

K

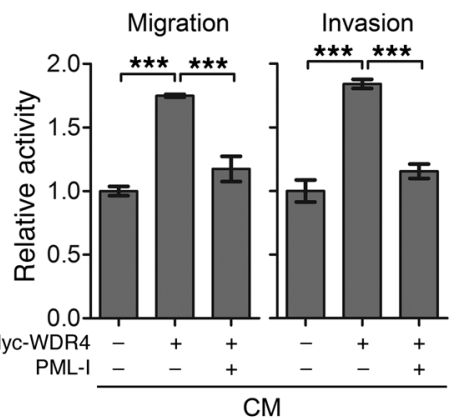

$\mathbf{L}$

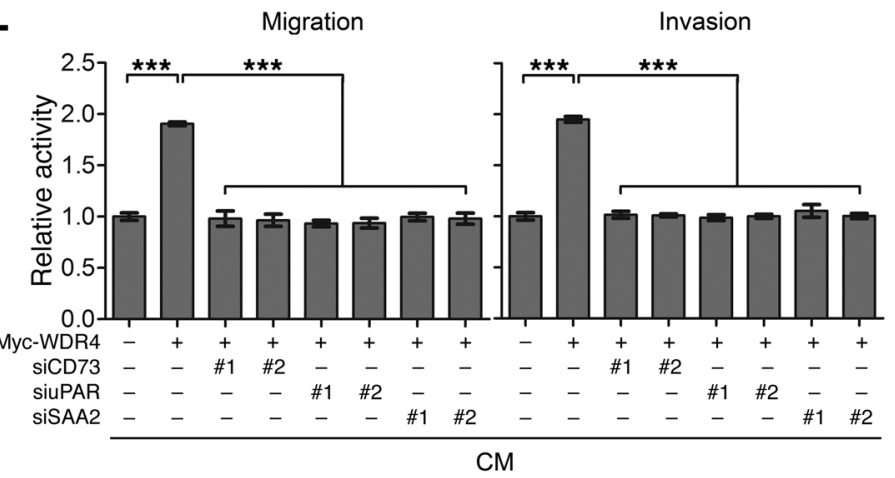

Figure 5. The WDR4/PML axis enhances lung cancer migration and invasion. (A) Western blot analysis of A549 cells stably expressing WDR4 shRNA and/or PML shRNA. (B) Migration and invasion activity of the cells in A. (C) Western blot analysis of A549 cells stably expressing WDR4 and/or PML-IV. (D) Migration and invasion abilities of the cells in C. (E) Western blot analysis of A549 cells stably expressing WDR4 and/or PML-I. (F) Migration and invasion assays of cells in $\mathbf{E}$. (C) Western blot analysis of WDR4-overexpressing A549 cells as in $\mathbf{C}$ and transfected with the indicated siRNAs. (H) Migration and invasion analyses of A549 cells stably expressing WDR4 and transfected with the indicated siRNAs. (I-L) Migration and invasion assays of parental A549 cells treated with CM derived from A549 stable lines, as in A, C, E, and G. Data represent the mean \pm SD; $n=3$ per group. ${ }^{* * *} P<0.001$, by 1 -way ANOVA with Tukey's post test.

The WDR4/PML axis promotes lung cancer migration and invasion. Next, we investigated the impact of the WDR4/PML axis on lung cancer migration and invasion. We established A549 and H1299 cell lines that stably expressed WDR4 shRNA or coexpressed WDR4 shRNA and PML shRNA (Figure 5A and Supple- mental Figure 5A). Remarkably, knockdown of WDR4 in A549 and H1299 cells suppressed cell migration and invasion, which were reversed by PML knockdown (Figure 5B and Supplemental Figure 5B). We made similar observations in the patient-derived primary lung cancer cells CL152 and CL141 (Supplemental Fig- 
A
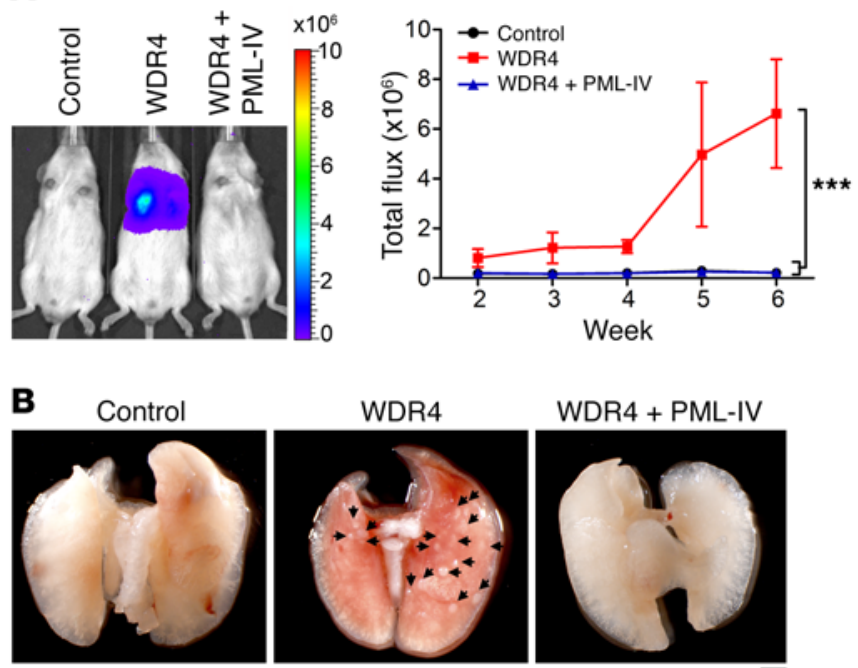

WDR4 + PML-IV
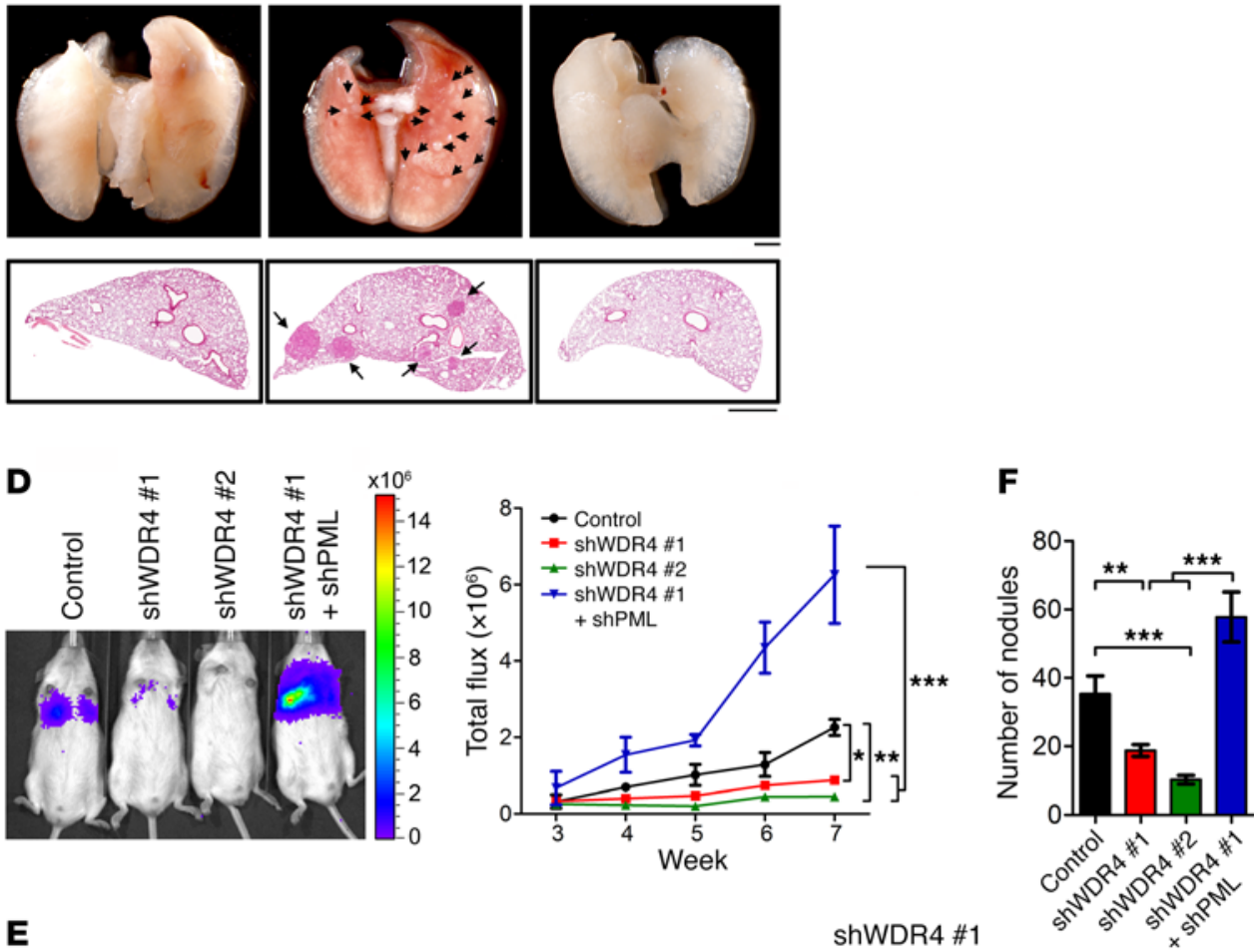

C

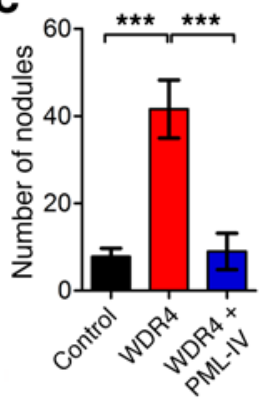

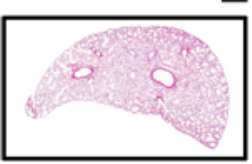

Week
E
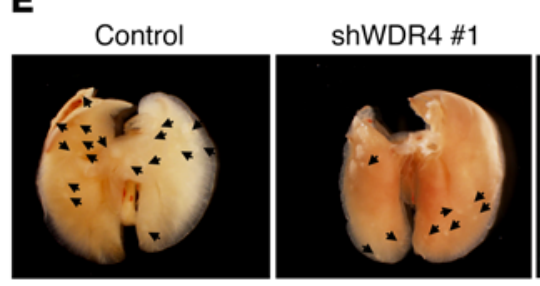

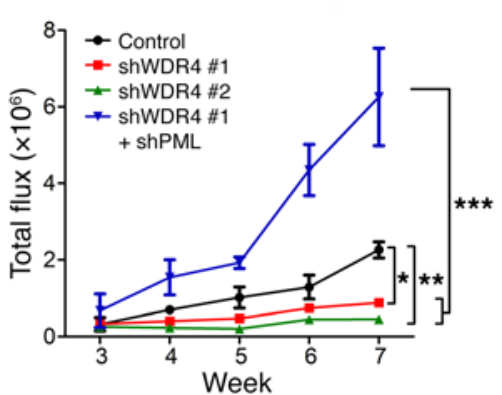

\section{F}

shWDR4 \# + ShPML
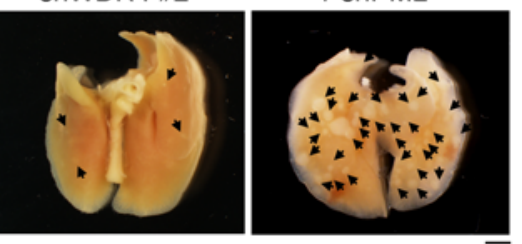
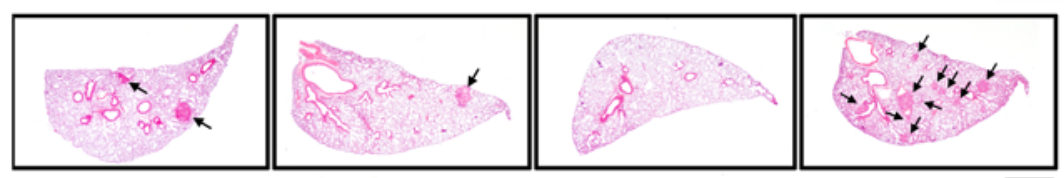

Figure 6. The WDR4/PML axis promotes lung cancer metastasis in xenograft mouse models. ( $A$ and $\mathbf{D}$ ) Bioluminescence analysis of lung metastasis derived from the indicated A549 cells (established in Figure 5). Representative images at week 6 (A) and week 7 (D) and the kinetics of metastasis at the indicated time points. Data in $\mathbf{A}$ and D represent the mean $\pm \mathrm{SD}$. ${ }^{* * *} P<0.001$ for week- 6 data ( $n=5$ per group) (A) and ${ }^{*} P<0.05,{ }^{*} P<0.01$, and ${ }^{* * *} P<0.001$ for week-7 data $(n=4$ per group) (D), all by 1-way ANOVA with Tukey's post test. (B and E) Lung metastasis and histological analysis of the lungs at week 6 (B) and week 7 (E). Nodules are indicated by arrows. Scale bars: $2 \mathrm{~mm}$ (top) and $500 \mu \mathrm{m}$ (bottom). (C and F) Number of metastatic nodules at the surface of the lungs at week 6 (C) and week 7 (F). Data represent the mean $\pm S D ; n=5$ per group $(\mathbf{C})$ and $n=4$ per group $(\mathbf{F}) .{ }^{* *} P<0.01$ and ${ }^{* * *} P<0.001$, by 1 -way ANOVA with Tukey's post test. ure 5, C-E). In reciprocal experiments, we established A549 and H1299 cells overexpressing WDR4 or WDR4 and PML-IV (Figure 5C and Supplemental Figure 5F). Compared with control cells and cells coexpressing WDR4 and PML-IV, WDR4-overexpressing cells showed greater migratory and invasive capabilities (Figure 5D and Supplemental Figure 5G). The migration- and invasion-promoting functions of WDR4 were also attenuated by overexpression of PML-I (Figure 5, E and F), the most abundant isoform of PML (37). Interestingly, knockdown of CD73, uPAR, or SAA2 each compromised the migration- and invasion-promoting effects of WDR4 overexpression or PML depletion (Figure 5, G and $\mathrm{H}$, and Supplemental Figure $5 \mathrm{H}$ ). These data support a role for the WDR4/PML axis in stimulating lung cancer migration and invasion and suggest that the induction of CD73/uPAR/SAA2 contributes to this migration and invasion.

Since the WDR4/PML axis induces the expression or activation of several secreted proteins such as SAA2, MMP2, and MMP9, which are known to promote migration and invasion, we tested the effect of CM taken from A549 and H1299 derivatives on the migration and invasion of parental A549 cells. CM 
A

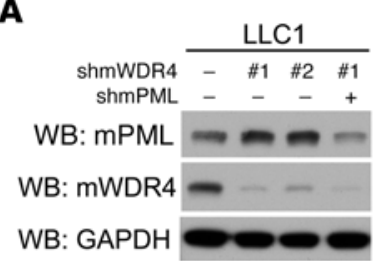

C
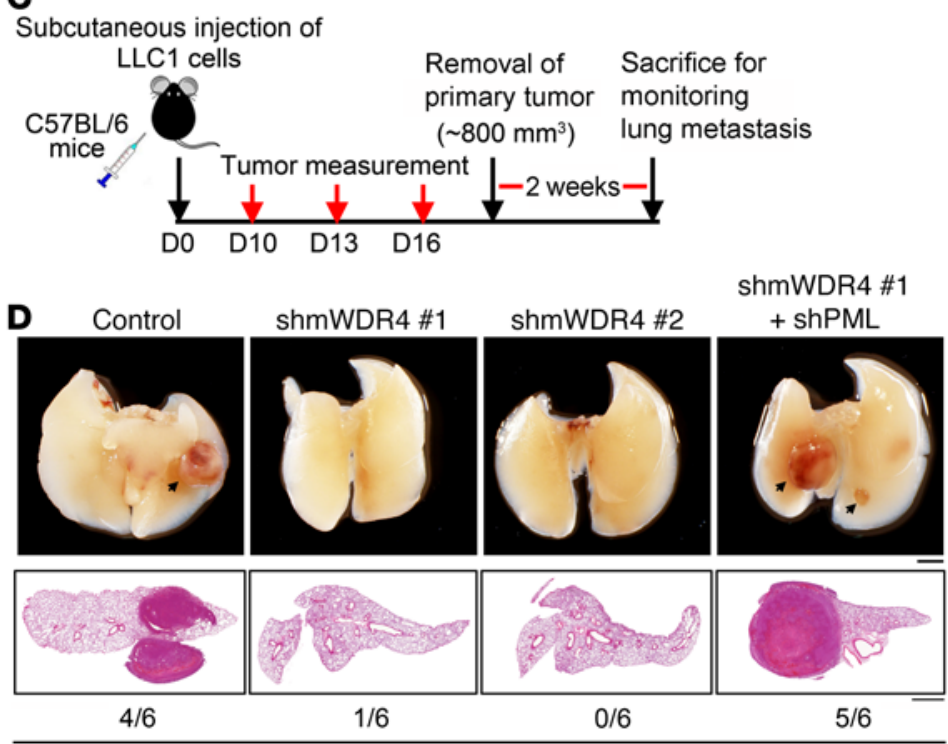

Mice with lung metastasis/ total mice

derived from WDR4-knockdown cells was less effective at stimulating cell migration and invasion compared with $\mathrm{CM}$ from control cells or WDR4/PML double-knockdown cells (Figure 5I and Supplemental Figure 5I). In a reciprocal set of experiments, CM from WDR4-overexpressing cells conferred higher migratory and invasive abilities than did CM from control cells or WDR4/ PML-IV- or WDR4/PML-I-coexpressing cells (Figure 5, J and K). Importantly, depletion of CD73, uPAR, or SAA2 each attenuated the paracrine effect of WDR 4 on the stimulation of migration and invasion (Figure 5L). These findings indicate that the WDR4/ PML axis stimulates lung cancer migration and invasion at least partly through the regulation of non-cell constituents of the tumor microenvironment and that CD73, uPAR, and SAA2 probably participate in this paracrine effect.

The WDR4/PML axis promotes lung cancer metastasis. To investigate the role of the WDR4/PML axis in lung cancer metastasis, we first undertook an experimental metastasis model by injecting A549 derivatives into the circulation of NOD/SCID mice. As revealed by bioluminescence analysis, cells overexpressing WDR4 showed a substantially greater potential to form metastases in lung compared with control, WDR4/PML-IV, or WDR4/PML-I cells (Figure 6A and Supplemental Figure 6A). Examination of lung for the presence of metastatic nodules confirmed the data obtained from bioluminescence analysis (Figure 6, B and C, and Supplemental Figure 6, B and C). Reciprocally, WDR4 knockdown in A549 cells and patient-derived primary lung cancer cells (CL152) suppressed lung metastasis, which was completely reversed by
Figure 7. The WDR4/PML axis promotes lung cancer metastasis in a syngeneic mouse model. (A) Western blot analysis of LLC1 cells stably expressing mWDR4 shRNAs and/or mPML shRNA. (B) BrdU incorporation assay for cells as in $\mathbf{A}$. Data represent the mean $\pm \mathrm{SD} ; n=4$ per group. NS, by 1-way ANOVA with Tukey's post test. (C) Schema for the tumor growth and metastasis assays of LLC1 derivatives inoculated in syngeneic mice. D, day. (D) Representative images of lung metastasis nodules (top), histological analysis of lung (middle), and the incidence of mice showing lung metastasis (bottom). Arrows indicate metastatic nodules. Scale bars: $2 \mathrm{~mm}$ (top) and $1 \mathrm{~mm}$ (middle).

PML knockdown (Figure 6, D-F, and Supplemental Figure 6, D-F). Thus, WDR4-mediated PML degradation plays a prometastatic role in lung cancer.

We further examined the prometastatic function of the WDR4/PML axis in lung cancer using an animal model to monitor the full range of metastatic properties including invasion, intravasation into the circulation, extravasation, and colonization at the metastatic site. To this end, we first established WDR4-knockdown and WDR4/PML double-knockdown lines in Lewis lung carcinoma (LLC1) cells, which were derived from lung adenocarcinoma in a C57BL/6 mouse. Knockdown of mouse WDR4 (mWDR4) upregulated mouse PML (mPML) in LLC1 cells (Figure $7 \mathrm{~A})$, indicating the existence of the WDR4/PML axis in the mouse. When we cultured the parental and knockdown cells in vitro, we did not observe a significant difference in proliferation (Figure 7B). We then inoculated these cells s.c. into C57BL/6 mice. To stimulate tumor metastasis (38) and to minimize the influence of the primary tumor size on metastatic progression, the primary tumors were removed when they reached $800 \mathrm{~mm}^{3}$ in size. Immunohistochemical analysis revealed lower expression levels of CD73, uPAR, and SAA2 in primary tumors derived from WDR4-knockdown cells than did tumors derived from control and WDR4/PML double-knockdown cells (Supplemental Figure 7A), indicating that the WDR4/PML axis similarly induces these 3 effectors in LLC1 cells. With this finding, we analyzed lung metastasis 2 weeks after removal of the primary tumors (Figure 7C). Importantly, WDR4-depleted LLC1 cells displayed a significant reduction in lung metastasis compared with control LLC1 cells. This metastasis-suppressive effect of WDR4 shRNA was reversed by PML shRNA (Figure 7D). Thus, our findings with this full-range metastasis model again demonstrated a critical role of the WDR4/PML axis in promoting lung cancer metastasis.

The WDR4/PML axis induces an immunosuppressive tumor microenvironment. Interestingly, we observed that WDR4 knockdown in LLC1 cells compromised the growth of primary tumors in the aforementioned syngeneic mouse model, and this effect was partially rescued by PML knockdown (Figure 8A). The difference in tumor growth correlated with the in vivo proliferation rate of the primary tumor cells, which was in sharp contrast to their similar proliferation rate observed in vitro (compare Figure 8B with Figure 7B). These findings suggest a role for the WDR4/PML axis in regulating the tumor microenvironment, thereby influencing the growth of primary tumors. We therefore investigated the stromal components that were affected by the WDR4/PML axis 
A

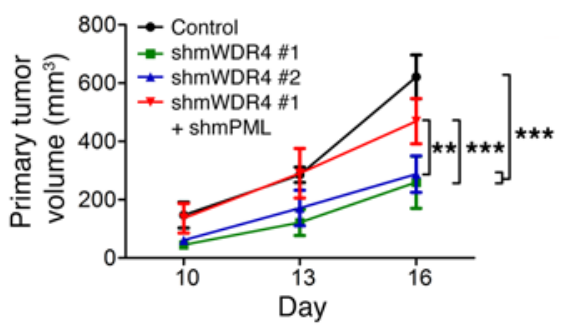

B

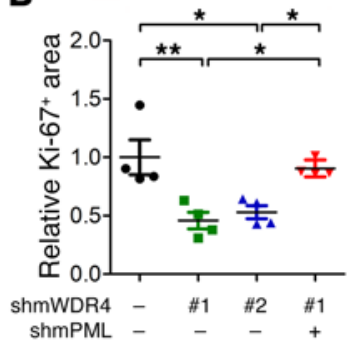

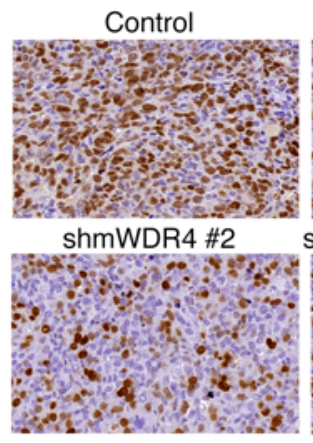

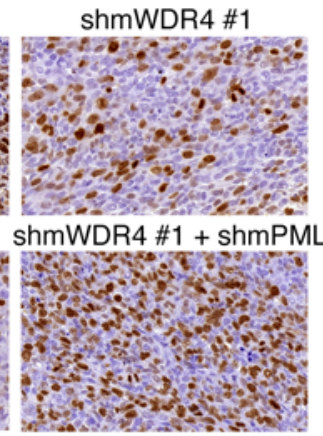

C

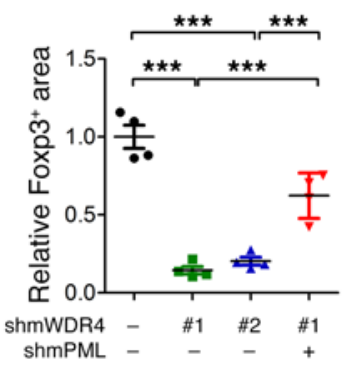

D

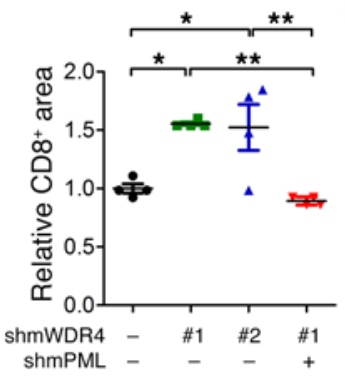

E

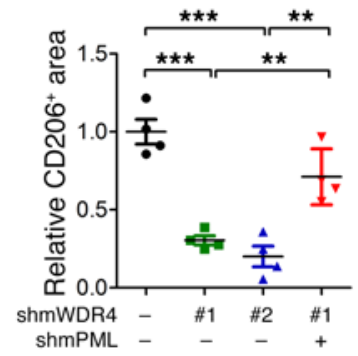

Control

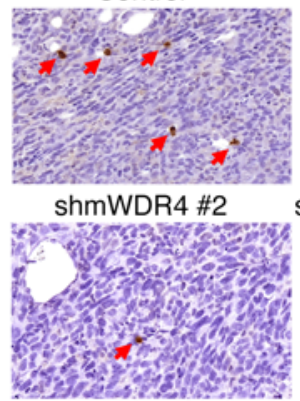

Control

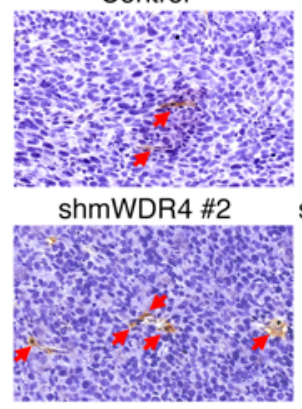

Control

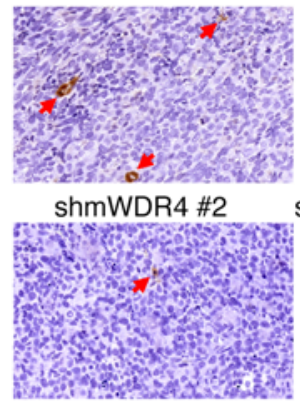

shmWDR4 \#1

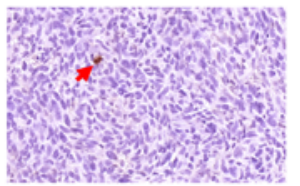

shmWDR4 \#1 + shmPML

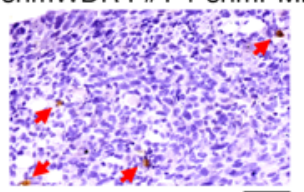

shmWDR4 \#1

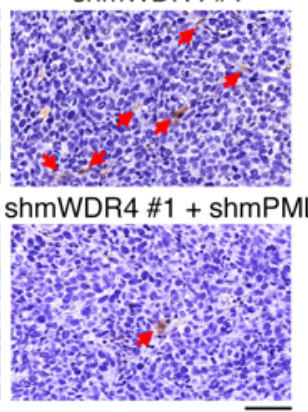

shmWDR4 \#1

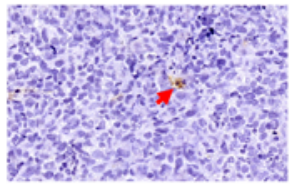

shmWDR4 \#1 + shmPML

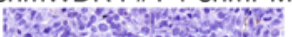

$\mathbf{F}$

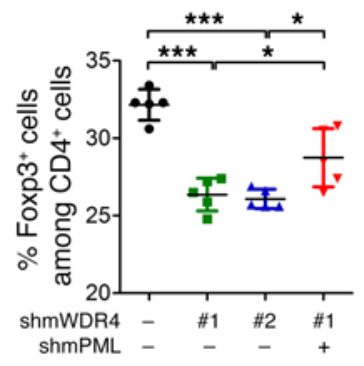

G

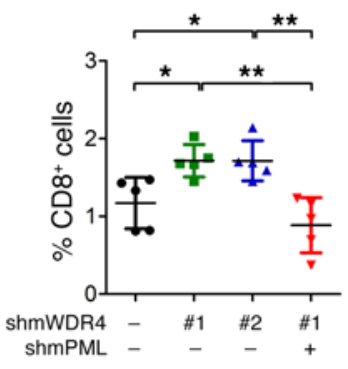

H

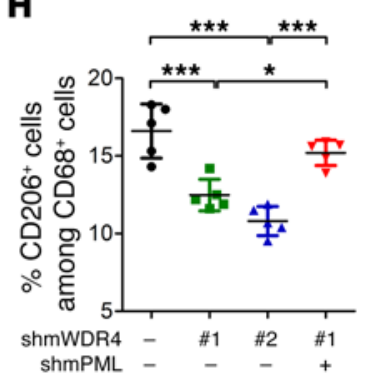

Figure 8. The WDR4/PML axis promotes lung cancer progression and induces an immunosuppressive tumor microenvironment in a syngeneic mouse model. (A) Primary tumor growth in the indicated groups. Data represent the mean $\pm S D ; n=6$ per group. ${ }^{* *} P<0.01$ and ${ }^{* * *} P<0.001$ for day-16 data, by 1 -way ANOVA with Tukey's post test. (B-E) Immunohistochemical analyses of Ki-67, Foxp3, CD8, and CD206 using primary tumors at the time of harvest (tumors were of similar size). Representative images are shown. Cells that stained positive are indicated by arrows. Data represent the mean $\pm S D ; n=4$ per group. ${ }^{*} P<0.05$, ${ }^{* *} P<0.01$, and ${ }^{* *} P<0.001$, by 1-way ANOVA with Tukey's post test. Scale bar: $50 \mu \mathrm{m}$. (F-H) Quantification of the flow cytometric data indicating the percentage of Foxp $3^{+}$cells among $\mathrm{CD} 4^{+} \mathrm{T}$ cells and $\mathrm{CD} 8^{+} \mathrm{T}$ cells and $\mathrm{CD} 206^{+} \mathrm{M2}$-like macrophages among $\mathrm{CD} 68^{+}$macrophages. In all experiments, the cells were first gated on $\mathrm{CD}_{4} 5^{+}$populations. Data represent the mean $\pm \mathrm{SD} ; n=5$ per group. ${ }^{*} P<0.05,{ }^{* *} P<0.01$, and ${ }^{* *} P<0.001$, by 1 -way ANOVA with Tukey's post test.

by performing immunohistochemical analysis to monitor tumorinfiltrating leukocytes. Although total $\mathrm{CD} 4^{+} \mathrm{T}$ cells and $\mathrm{NK} 1.1^{+}$ pan-NK cells were not altered (Supplemental Figure 7, B and C), the number of intratumoral Foxp $3^{+}$Tregs was reduced in tumors derived from WDR4-knockdown cells, and this was accompanied by an increase in the number of $\mathrm{CD}^{+} \mathrm{T}$ cells (Figure $8, \mathrm{C}$ and $\mathrm{D}$ ). Additionally, CD68 ${ }^{+}$pan-macrophages and CD206 ${ }^{+} \mathrm{M} 2$-like (protumor) macrophages were moderately and markedly reduced in 

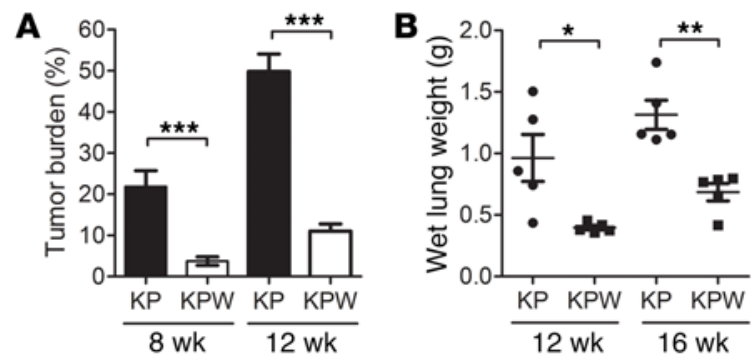

$\mathbf{E}$
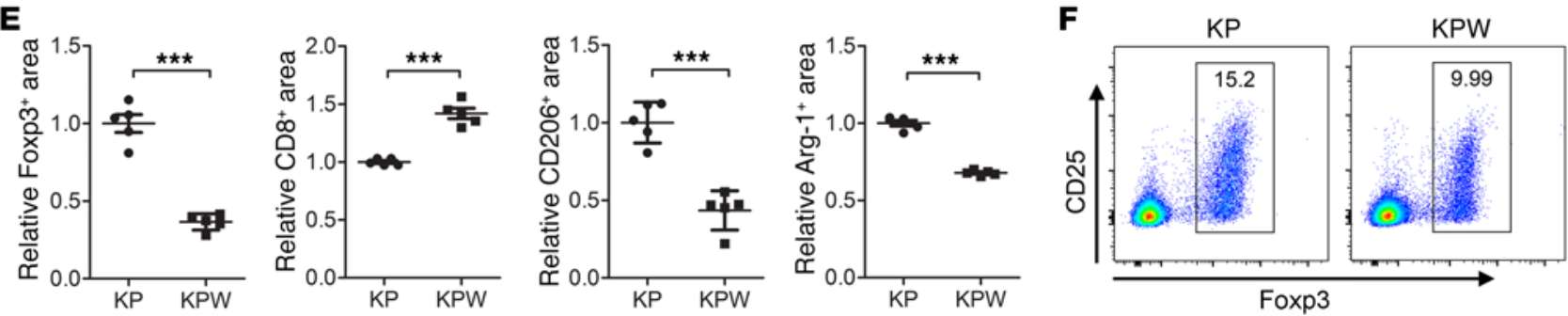

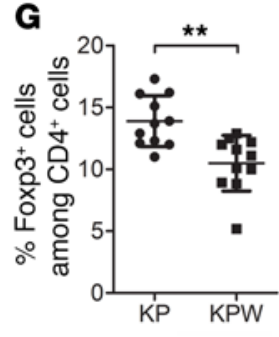

J

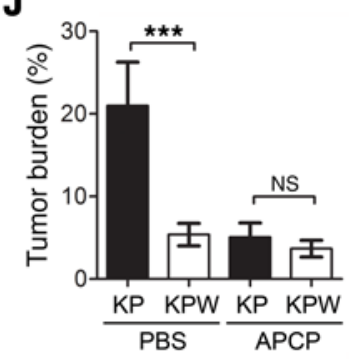

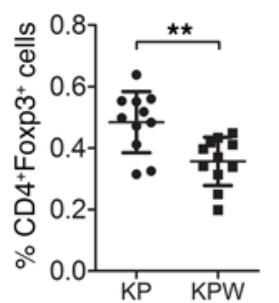

K

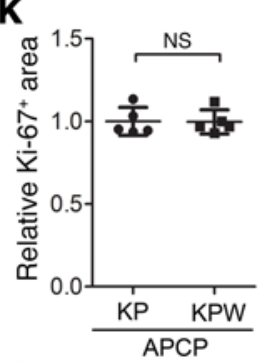

H

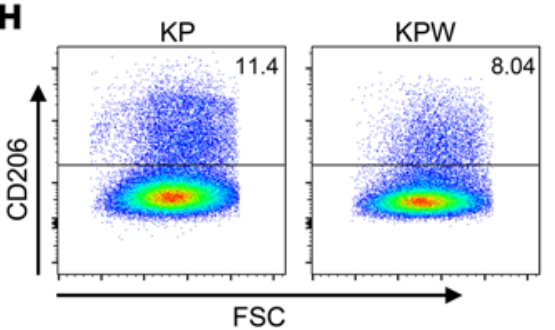

$\mathbf{L}$

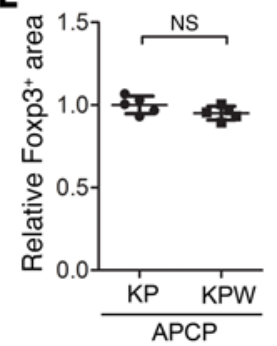

$\mathbf{F}$

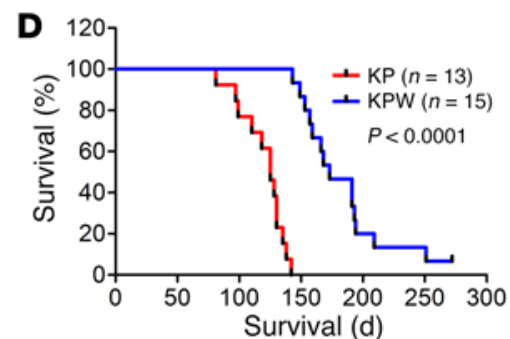

I

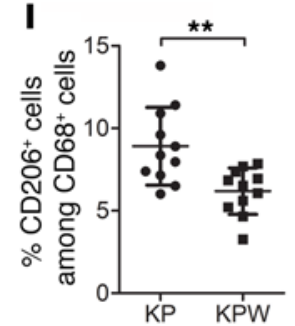

Figure 9. WDR4 ablation suppresses p53 deficiency and Kras-driven lung tumorigenesis by inhibiting CD73-dependent immunosuppressive functions. (A and B) Quantification of tumor burden and lung weights for KP and KPW mice at the indicated time points after Ad-Cre administration. Data represent the mean $\pm \mathrm{SD} ; n=5$ per group. ${ }^{*} P<0.05$, ${ }^{* *} P<0.01$, and ${ }^{* *} P<0.001$, by 2 -tailed Student's $t$ test. (C, E, $\mathbf{K}$, and $\mathbf{L}$ ) Quantification of immunohistochemical data for Ki-67+ cells and the indicated immune cells in lung tumors from KP and KPW mice 8 weeks after tumor induction, with or without APCP treatment. Representative IHC images are shown in Supplemental Figure 8, C, E, H, and I. Data represent the mean \pm SD; $n=5$ per group. ${ }^{* * *} P<0.001$, by 2-tailed Student's $t$ test. (D) Kaplan-Meier survival curves for KP mice $(n=13)$ and KPW mice $(n=15)$. The time of Ad-Cre administration is defined as day

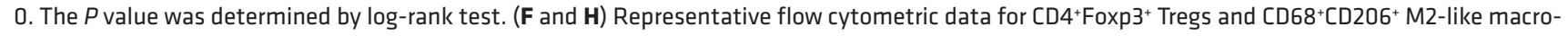
phages in the lungs of KP and KPW mice 8 weeks after Ad-Cre administration. Cells were gated on CD4+ populations (F) or CD68 $8^{+}$populations (H). Numbers indicate the percentages among total CD4+ cells (F) or CD68 ${ }^{+}$cells $(\mathbf{H})$ in the lung. FSC, forward scatter. ( $\mathbf{G}$ and $\left.\mathbf{I}\right)$ Quantification of the flow cytometric data for the percentages of Foxp3 ${ }^{+}$cells among $\mathrm{CD}^{+} \mathrm{T}$ cells, $\mathrm{CD4}^{+} \mathrm{Foxp3}^{+}$cells among total analyzed lung cells, and CD206+ $\mathrm{M2}^{-}$-like macrophages among CD68 ${ }^{+}$macrophages. Data represent the mean $\pm \mathrm{SD} ; n=11$ per group. ${ }^{* *} P<0.01$, by 2-tailed Student's $t$ test. (J) Quantification of the tumor burden in KP and KPW mice 8 weeks after administration of Ad-Cre, with or without APCP treatment. Data represent the mean $\pm S D ; n=5$ per group. ${ }^{* * *} P<0.001$, by 2-tailed Student's $t$ test.

tumors derived from WDR4-knockdown cells, respectively (Supplemental Figure 7D and Figure 8E). These WDR4-knockdown effects were all reversed by WDR4/PML double knockdown. Consistent with these immunohistochemical data, our flow cytometric analysis of the primary tumors also revealed a decrease in Tregs and M2-like macrophages and an increase in $\mathrm{CD}^{+} \mathrm{T}$ cells in tumors derived from WDR4-knockdown cells compared with tumors derived from control and WDR4/PML double-knockdown cells (Figure 8, F-H, and Supplemental Figure 7E). Our findings thus uncovered a role for the WDR4/PML axis in fostering an immunosuppressive tumor microenvironment.

WDR4 acts through CD73 to suppress antitumor immunity. To further examine the role of WDR4 in lung cancer progression and tumor microenvironment remodeling, we used a genetically engineered mouse model (GEMM). To this end, LSL-Kras ${ }^{G 12 D /+} p 53^{f / f l}$ $W d r 4^{f / f l}$ mice (referred to hereafter as KPW mice) were generated 


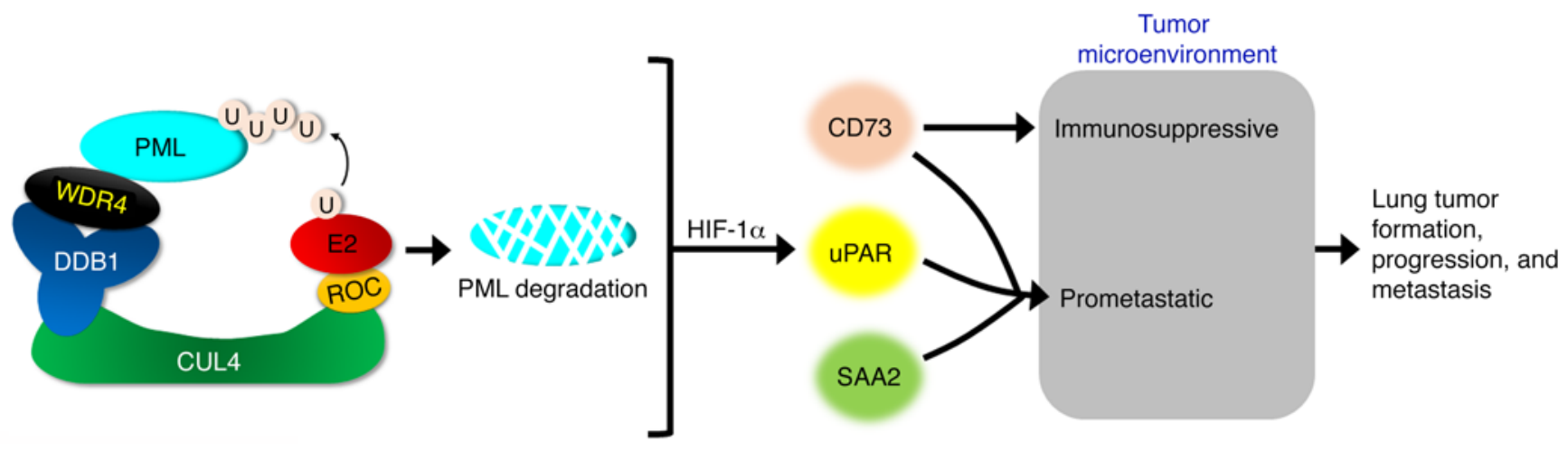

Figure 10. Model for WDR4-mediated PML degradation and its impacts on the tumor microenvironment. WDR4-based CRL4 ubiquitin ligase is responsible for PML ubiquitination (U) and destruction. The WDR4-mediated PML degradation pathway acts through HIF-1 $\alpha$ to induce the expression of CD73, UPAR, and SAA2, which function in coordination to foster an immunosuppressive and prometastatic lung tumor microenvironment, thereby potentiating lung tumor formation, progression, and metastasis.

by crossing Wdr4 ${ }^{f / f l}$ mice (39) with $L S L-\operatorname{Kras}^{G 12 D /+} p 53^{\text {fl/fl }}$ mice (referred to hereafter as KP mice), a well-established GEMM for human lung adenocarcinoma (40). Lung tumorigenesis was induced by intratracheal administration of Cre-expressing adenovirus (Ad-Cre), which led to the simultaneous expression of oncogenic $\mathrm{Kras}^{\mathrm{G12D}}$ and deletion of transformation-related protein 53 (Trp53) and Wdr4. Lung tumors derived from KPW mice expressed only trace amounts of WDR4, which were presumably derived from stromal cells (Supplemental Figure 8A). Furthermore, KPW lung tumors expressed higher levels of PML and lower levels of CD73, uPAR, and SAA2 than did KP lung tumors (Supplemental Figure 8A). Importantly, the KPW mice showed significant and persistent reductions in lung tumor burden and lung weight, which correlated with a decrease in lung tumor cell proliferation (Figure 9, A-C, and Supplemental Figure 8, B and C). Pathological analysis revealed a lower number of total lung lesions in KPW lungs than was observed in KP lungs, especially for adenoma and adenocarcinoma (Supplemental Figure 8D), suggesting that WDR4 deficiency impairs lung tumor formation and progression. Accordingly, the KPW mice had significantly prolonged survival (Figure 9D). These findings indicate a lung tumor-promoting effect of WDR4 in this GEMM.

Consistent with our observations from the syngeneic mice model, lung tumors derived from KPW mice contained fewer Foxp $^{+}$Tregs and CD206 ${ }^{+}$M2-like macrophages and more CD8 ${ }^{+}$ $\mathrm{T}$ cells than did tumors from KP mice (Figure 9E and Supplemental Figure 8E). A reduction of M2-like macrophages in KPW lung tumors was also observed by examining another marker, arginase 1 (Arg-1) (Figure 9E and Supplemental Figure 8E). Furthermore, flow cytometric analysis confirmed decreased CD $4^{+}$Foxp $3^{+}$Tregs in the KPW mouse lungs, but not in the local draining mediastinal lymph nodes (Figure 9, F and G, and Supplemental Figure 8F). We also observed a decreased ratio of $\mathrm{CD}^{2} \mathrm{O6}^{+} \mathrm{M}$ 2-like macrophages to total $\left(\mathrm{CD}^{+} 8^{+}\right)$macrophages in KPW mouse lungs compared with the ratio in KP mouse lungs, suggesting an inhibition of M2 polarization by WDR4 deficiency (Figure 9, H and I). Notably, CD73 is known to promote Treg accumulation and M2 macrophage differentiation in the tumor microenvironment $(29,41,42)$, and Tregs can in turn suppress CD ${ }^{+} \mathrm{T}$ cells $(43,44)$. We therefore tested whether the differences in tumorigenesis and tumor microenvironment regulation between KP and KPW mice are due to a differential expression of CD73 in their lung lesions. In keeping with this notion, systematic treatment with the CD73 inhibitor $\alpha$, $\beta$-methylene adenosine- 5 '-diphosphate (APCP) abrogated the differences in tumor burden and tumor cell proliferation seen in KP and KPW mice (Figure 9, J and K, and Supplemental Figure 8, G and $\mathrm{H}$ ). Furthermore, APCP-treated KP and KPW mice had comparable numbers of Tregs, $\mathrm{CD} 8^{+} \mathrm{T}$ cells, and M2-like macrophages in their lung tumors (Figure 9L and Supplemental Figure 8I). Thus, our study revealed a key role of WDR4 in lung tumorigenesis and progression in GEMMs, a function that is at least partly mediated through CD73-dependent immunosuppressive effects.

\section{Discussion}

In this study, we identify WDR4 as a substrate adaptor of CRL4. Our findings are consistent with a previous study that identified WDR4 as one of the proteins stabilized by CUL4 inhibition (45). Importantly, CRL $4^{\mathrm{WDR} 4}$ catalyzes the polyubiquitination of the tumor suppressor PML, resulting in its proteasomal degradation. We further show that high WDR4 expression frequently occurs in lung cancer and correlates with PML downregulation and poor patient survival, which supports the clinical relevance of this PML degradation pathway to lung malignancy. The mechanism for WDR4 upregulation in lung cancer is currently unknown. Since amplification of the WDR4 genomic locus is rare in lung cancer ( $0.4 \%$ revealed by the cBioportal for Cancer Genomics database, http://www.cbioportal.org/), this upregulation probably occurs at a transcriptional or posttranscriptional level. Interestingly, amplification of the CUL $4 A$ gene has been observed in a small subset of patients with lung cancer (46), suggesting an alternative mechanism for PML downregulation in lung cancer.

The functional impact of WDR4-mediated PML degradation on lung tumor formation and progression has been uncovered by the identification of a set of cell-surface or secreted proteins that are upregulated or activated by WDR4 overexpression or PML depletion. Among them, uPAR, SAA2, MMP2, and MMP9 can each induce ECM remodeling through direct or indirect mechanisms $(31,32,47,48)$. Although PML was previously reported to suppress 
the expression of MMP2 and integrin $\beta 1$ in certain cell types (10, 49), their expression was not significantly induced by the WDR4/ PML axis in our cell system. Instead, our study suggests that the WDR4/PML axis acts through UPAR and SAA2 to influence the activity of integrin and MMPs. Additionally, the 3 effectors of the WDR4/PML axis, i.e., CD73, SAA2, and UPAR, can each function as an ECM adhesion molecule to influence the adhesion of tumor cells $(29,31,32,48)$. Thus, the WDR4/PML axis could elicit multifaceted mechanisms, such as the promotion of ECM remodeling and degradation and enhancement of cell adhesion, to influence tumor cell motility and invasiveness. Accordingly, the WDR4/ PML axis promotes lung tumor cell migration and invasion, and CD73, uPAR, and SAA2 each participates in these effects. Since these effects are recapitulated using CM derived from tumor cells, the non-cell constituents of the tumor microenvironment, such as ECM-degrading enzymes and other soluble factors, should play a role in the migratory and invasive functions of the WDR4/PML axis. In line with these migratory and invasive functions, the PML degradation pathway enhances tumor metastasis, as assessed by 2 animal models of metastasis that monitor the partial and full process of the tumor invasion-metastasis circuit, respectively. Thus, our study identifies a profound role of the WDR4/PML axis in lung cancer metastasis, one that is at least partly mediated through a set of effectors including CD73, uPAR, SAA2, and MMPs.

Besides the non-cell constituents, the cell constituents of the tumor microenvironment also play a crucial role in tumor progression. Although the role of PML in stimulating IFN- $\beta$ expression to contribute to antiviral responses was previously reported (50), its involvement in antitumor immune surveillance has not been explored. In the lung cancer cell system used in this study, IFNB mRNA expression was below the detection limit (data not shown), indicating a context-dependent effect. Nevertheless, we identified CD73 as an effector of the WDR4/PML axis. By converting extracellular AMP to adenosine, CD73 suppresses antitumor immune cells and enhances regulatory immune cells to establish an immunosuppressive tumor microenvironment (29). Accordingly, we found that the WDR4/PML axis increases intratumoral Tregs and M2-like macrophages and decreases $\mathrm{CD}^{+} \mathrm{T}$ cells in a syngeneic mouse model, which coincides with an increased proliferation of tumor cells in vivo. In line with these findings, WDR4 deficiency in a GEMM of lung cancer (KP model) decreases intratumoral Tregs and M2-like macrophages but increases $\mathrm{CD}^{+} \mathrm{T}$ cells, thereby suppressing lung tumor formation and progression to prolong survival. Since the phenotypes of WDR4 ablation in this GEMM resemble those in the syngeneic mouse model, in which PML knockdown rescues the effects of WDR4 knockdown, we postulate that PML stabilization accounts for a major mechanism of the tumor-suppressive effects of WDR4 deficiency seen in this GEMM. In support of this notion, blockage of CD73, an effector of the WDR4/PML axis, completely inhibits the tumor promotion and immunosuppressive functions of WDR4. These findings strongly support a major contribution of CD73-mediated adenosinergic signaling to the phenotypes of WDR4 deficiency observed in the GEMM, even though we cannot completely rule out the involvement of other targets or partners of WDR4 or PML. Of note, our data derived from the GEMM also suggest the existence of a cooperative function of the WDR4/PML axis with Kras mutation and $p 53$ deficiency in lung tumorigenesis.
Recent studies have revealed a 2-way interaction between tumor cells and immune cells in the tumor microenvironment. On one hand, activation of tumor-intrinsic oncogenes such as $\beta$-catenin (CTNNB1) (51), STAT3 $(52,53)$, enhancer of zeste 2 polycombrepressive complex 2 subunit (EZH2), and DNA methyltransferase 1 (DNMT1) (54) and loss of function of the tumor suppressor p53 (55) have been shown to contribute to immune exclusion by influencing the production of cytokines and/or chemokines by tumor cells. On the other hand, immune cells in the tumor microenvironment can not only induce cancer dormancy (56) or foster immune evasion (57), but also promote tumor stemness and metastasis-related traits $(58,59)$. The immune-suppressive effects of PML degradation identified in this study have expanded the list of tumor-intrinsic oncogenic programs with immunemodulatory functions. Future studies will elucidate whether these immune-modulatory functions can trigger a feedback effect to further enhance the malignant traits of tumor cells.

In summary, we identify what we believe to be a new PML ubiquitination and degradation pathway, manifest its hyperactivation in lung cancer, and elucidate its significance in remodeling both non-cell and cell constituents of the tumor microenvironment to promote lung cancer growth and metastasis (Figure 10). In addition to uncovering previously unreported prometastatic targets, our study has expanded the pleiotropic tumor-promoting mechanisms of PML degradation to include the establishment of an immunosuppressive tumor microenvironment, which implies potential immune-modulatory approaches for treating lung cancer with aberrant PML degradation.

\section{Methods}

Cell culture. 293T, 293FT, H1299, H460, and A549 cells were obtained from ATCC. CL1-O and LLC1 cells were provided by Pan-Chyr Yang (National Taiwan University) and Muh-Hwa Yang (National YangMing University, Taipei, Taiwan). 293T, 293FT, H1299, CL1-0, and A549 cells were cultured in DMEM. H460 cells were maintained in RPMI 1640 medium. LLC1 cells were cultured in DMEM high-glucose medium. All media were supplemented with $1 \%$ penicillin/streptomycin (Gibco, Thermo Fisher Scientific) and 10\% FBS. The primary human lung cancer cells CL141 and CL152 were established previously (60) by isolating cells from the pleural effusion of patients with nonsmall-cell lung cancer at the National Taiwan University Hospital (Taipei, Taiwan) and were cultured in RPMI 1640 medium supplemented with sodium pyruvate and $10 \%$ FBS.

Plasmids. Plasmids encoding Myc-ubiquitin, His-ubiquitin, MycCUL3, Myc-ROC1, Flag-PML-I, and Flag-PML-IV were described previously (18). WDR4 cDNA (39) was subcloned to pRK5 with a Myc- or V5-tag, and WDR4 mutants were generated by site-directed mutagenesis. All CUL-DN mutants were generated by site-directed mutagenesis from WT constructs obtained from Hsueh-Chi Sherry Yen (Institute of Molecular Biology, Academia Sinica, Taipei, Taiwan). pcDNA3HA-DDB1 (plasmid no. 19909), pcDNA3-Myc3-CUL4A (plasmid no. 19951) and pcDNA3-Myc3-CUL4B (plasmid no. 19922) were purchased from Addgene. For the establishment of lentivirus-based constructs, Myc-WDR4, Flag-PML-I, and Flag-PML-IV were subcloned to pLAS3w.Pneo or pLAS3w.phyg.

Antibodies and reagents. The antibody against mouse WDR4 was described previously (39). Other antibodies used in this study are 
listed in Supplemental Table 1. MG132 was purchased from Calbiochem. CHX was obtained from Sigma-Aldrich.

RT-qPCR. Total RNA was extracted from cells using TRIzol Reagent (Invitrogen, Thermo Fisher Scientific) or a NucleoSpin RNA Kit (Macherey-Nagel) and quantified by NanoDrop (Thermo Fisher Scientific). Reverse transcription was performed using an iScript cDNA Synthesis Kit (Bio-Rad). For qPCR analysis, a LightCycler 480 SYBR Green I Master Kit (Roche) was used, and amplification was performed on a LightCycler 480 System. To normalize for cDNA loading, GAPDH or ACTB was used as an internal control. The PCR primers used in this study are listed in Supplemental Table 2.

RNA interference and lentivirus transduction. Lentivirus-based shRNA constructs were obtained from the National RNAi Core Facility in Taiwan, and lentivirus generation and transduction were described previously (18). Various siRNAs were obtained from Dharmacon. The target sequence for each shRNA and siRNA is listed in Supplemental Table 3.

Western blotting, immunoprecipitation, and GST pull-down. Cell extraction was performed with RIPA lysis buffer containing $50 \mathrm{mM}$ Tris (pH 8.0), $150 \mathrm{mM} \mathrm{NaCl}, 1 \% \mathrm{NP}-40,1 \%$ sodium deoxycholate, $0.1 \%$ SDS, $1 \mathrm{mM}$ phenylmethyl sulphonyl fluoride, $1 \mu \mathrm{g} / \mathrm{ml}$ aprotinin, and $1 \mu \mathrm{g} / \mathrm{ml}$ leupeptin. Immunoprecipitation using cell lysates containing equal amounts of proteins was performed as described previously (61). For GST pull-down, recombinant Flag-PML-I was purified from baculovirus with anti-Flag agarose beads and eluted with Flag-peptide. GST-WDR4 or GST was immobilized on glutathione-sepharose beads and incubated with the Flag-PML-I in the binding buffer containing $50 \mathrm{mM}$ Tris (pH 7.5), $150 \mathrm{mM} \mathrm{NaCl}$, and 1\% NP-40. The beads were washed, and bound proteins were analyzed by Western blotting.

Ubiquitination assays. In vitro ubiquitination of baculovirally purified PML-I using a CRL4 ${ }^{\mathrm{WDR} 4}$ E3 ligase complex purified from transfected 293T cells was performed following a previously described protocol (18). For in vivo ubiquitination, cells transfected with various constructs, together with Myc-ubiquitin or His-ubiquitin, were treated with MG132 for 16 hours and lysed by RIPA buffer or under denaturing conditions, respectively. Ubiquitination was analyzed by immunoprecipitation as previously described (18).

Microarray. cDNA microarray was conducted by Phalanx Biotech using the Human OneArray platform. GO analysis was performed using the Database for Annotation, Visualization and Integrated Discovery (DAVID) Bioinformatics Resources 6.7 (https://david.ncifcrf. gov/). The data sets of the cDNA microarray for WDR4 versus control and for PML siRNA versus control were deposited in the NCBI's GEO database (GEO GSE83499).

CM preparation. For preparation of the CM, cells $\left(7 \times 10^{6}\right.$ for the MMP assay and $1.2 \times 10^{6}$ for Western blotting) were cultured in serumfree medium for 48 hours. Cell viability was ascertained by a trypan blue dye exclusion assay and was greater than 95\%. The media were collected and concentrated with Amicon Ultra centrifugal filter units.

MMP activity assay. MMP2 and MMP9 activity was assayed using a QuickZyme Human Activity MMP2 Assay Kit (QuickZyme Bioscience) and an Amersham MMP9 Biotrack Activity Assay System (GE Healthcare), respectively. In brief, concentrated $\mathrm{CM}$ was added onto precoated wells. After incubation at $4^{\circ} \mathrm{C}$ overnight, detection reagent was added, and the plate was read at $405 \mathrm{~nm}$ to obtain values at $t=$ 0 hours. The plate was then incubated at $37^{\circ} \mathrm{C}$ for 6 hours to obtain values at $t=6$ hours. Endogenous MMP activity was calculated by subtracting the values at $t=0$ hours from those at $t=6$ hours and normalized to control.

Migration and invasion assays. For Transwell migration and invasion assays, the underside of a Transwell polycarbonate membrane (8- $\mu \mathrm{m}$ pore size) (EMD Millipore) was coated with $15 \mu \mathrm{g} / \mathrm{ml}$ collagen. For the migration assay, $4 \times 10^{4}$ cells resuspended in serum-free medium containing $1 \%$ BSA were plated onto the upper chamber, and the medium containing $1 \%$ BSA and $20 \%$ FBS was added to the lower chamber. For the invasion assay, the upper side of a Transwell membrane was precoated with Matrigel, and $6 \times 10^{4}$ cells were plated onto the upper chamber. For assays using CM, 4 -fold concentrated $\mathrm{CM}$ was added to the lower chamber. Cells were incubated at $37^{\circ} \mathrm{C}$ for 6 hours (for the migration assay) or 18 hours (for the invasion assay). To distinguish the migration/invasion effect from the proliferation effect, the same number of cells were seeded onto a regular culture plate. At the end of incubation, cells that had migrated onto the lower membrane surface of the Transwell plate were fixed with $4 \%$ formaldehyde, stained with DAPI, counted, and normalized to the number of cells appearing in the regular plate.

Proliferation assay. Cell proliferation was assayed using a Cell Proliferation ELISA BrdU Kit (Roche). In brief, $3 \times 10^{3}$ cells were seeded in each well of a 96-well plate, and BrdU was added to the CM 18 hours after plating. Cells were incubated for 1 hour before harvesting.

Animal experiments. For the experimental metastasis model, cells tagged with luciferase were resuspended $\left(1 \times 10^{6}\right.$ cells in $\left.100 \mu \mathrm{l} \mathrm{PBS}\right)$ and injected into the tail vein of 6-week-old male NOD/SCID mice (BioLASCO Taiwan Co.). Lung metastasis was monitored by bioluminescence imaging using a PerkinElmer in vivo imaging system (IVIS) and by histological analysis.

For the syngeneic mouse model, $2.5 \times 10^{5} \mathrm{LLC} 1$ cells were inoculated into the s.c. dorsal area of 6-week-old male C57BL/6 mice (National Laboratory Animal Center, Taipei, Taiwan). The size of primary tumors was measured regularly after tumor inoculation. Primary tumors were surgically removed when they reached approximately $800 \mathrm{~mm}^{3}$ in size, and lungs were harvested 2 weeks later.

For the GEMM, KPW mice were generated by crossing $W d r 4^{f / f l}$ mice (39) with KP mice (Mouse Models of Human Cancer Consortium), which were maintained on a mixed 129Sv/C57BL/6 background. For lung tumor induction, intratracheal inhalation of 6- to 8-week-old male and female mice with Ad-Cre viruses (Gene Transfer Vector Core, University of Iowa) was performed according to a previously reported protocol (40). APCP (Sigma-Aldrich) was injected i.p. at a daily dose of 20 $\mathrm{mg} / \mathrm{kg}$ for 10 days, starting on 1 day after Ad-Cre administration, and then at a dose of $10 \mathrm{mg} / \mathrm{kg}$ at 2-day intervals until sacrifice.

Histology and immunohistochemical analyses. Lungs were removed from the mice after heart perfusion, fixed in $4 \%$ paraformaldehyde for 2 days, and then transferred to $50 \%$ ethanol for the preparation of paraffin-embedded sections. For histological analysis, sections were stained with H\&E. Tumor burden (tumor area to total lung area) was quantified with PatternQuant module (3DHISTECH Ltd.). For immunohistochemical analysis, antigen retrieval was performed by boiling in target retrieval solution (S1700; Dako) with a pressure cooker for 20 minutes (for mouse tissues) or by heat denaturation of paraffinembedded sections with $10 \mathrm{mM}$ sodium citrate (pH 6.0) for 30 minutes. Endogenous peroxidase activity was blocked by incubation in $3 \%$ $\mathrm{H}_{2} \mathrm{O}_{2}$ at room temperature for 20 minutes. The sections were blocked with PBS containing $10 \%$ goat serum and incubated with various anti- 
bodies at $4^{\circ} \mathrm{C}$ for overnight. The bound antibody was detected by the Super-Picture Polymer Detection Kit (Invitrogen, Thermo Fisher Scientific). The counterstaining was performed with hematoxylin. WDR4 and PML expression in human specimens was scored semiquantitatively as high or low on the basis of staining intensity and percentage of cells that stained positive. For the quantification of tumor-infiltrating leukocytes, whole lung images were acquired using Pannoramic 250 FLASH II software. The ratio of immunohistochemical-positive area to hematoxylin-positive area in 24 tumor regions randomly selected by the Pannoramic Viewer was analyzed by ImageJ software (NIH).

Human specimens. The multitissue microarray (MTU951) was obtained from Biomax Inc. Lung cancer tissue specimens were obtained from the National Cheng Kung University Hospital. Detailed clinicopathological characteristics of the enrolled patients are provided in Supplemental Table 4.

Flow cytometry. For flow cytometric analysis of mouse lung cells, blood was removed from the lungs by heart perfusion. The resected mouse lungs were washed with PBS twice to remove residual blood and were mechanically dissociated with surgical scissors. To generate single cells, lungs were digested for 40 minutes at $37^{\circ} \mathrm{C}$ in HBSS containing collagenase type I $(0.5 \mathrm{mg} / \mathrm{ml})$, collagenase type IV $(0.5 \mathrm{mg} /$ $\mathrm{ml})$, DNase I (40 U/ml), hyaluronidase $(0.2 \mathrm{mg} / \mathrm{ml})$, and $\mathrm{CaCl}_{2}(5 \mathrm{mM})$. The tumor suspension was strained using a $70-\mu \mathrm{m}$ cell strainer, and red blood cells were lysed with ammonium chloride potassium (ACK) lysing buffer. Single-cell suspensions were preincubated with antiCD16/32 Fc receptor-blocking antibody (clone 93; eBioscience) and stained with the respective antibodies. Foxp3 staining was performed according to the manufacturer's instructions. Fixable Viability Dye eFluor 780 (eBioscience) was used to exclude dead cells from analysis. FACS analysis was performed with the LSRII Flow Cytometer (BD Biosciences) and analyzed with FlowJo 10.0.7 software.

Bioinformatics. TCGA level 3 RNAseqV2 data, along with the clinical data (https://wiki.nci.nih.gov/display/TCGA/RNASeq+Version+2) released on March 4, 2015, were downloaded automatically using a modified R package, TCGA-Assembler. Additionally, microarray data from human lung cancer patients were retrieved from the NCBI's GEO databases. The data were normalized and transformed as $\log _{2}$ values. The analysis of lung cancer patient survival was conducted through the ProGgeneV2 Prognostic Database (http://watson.compbio.iupui. edu/chirayu/proggene/database/index.php).

Statistics. A 2-tailed, unpaired $t$ test was used for comparisons between 2 groups, and ANOVA was used for multigroup comparisons. Fisher's exact test was applied for analysis of the association with clinicopathological data. A Kaplan-Meier estimation and log-rank test were used to compare survival differences. A $P$ value of less than 0.05 was considered statistically significant.

Study approval. All studies using human tissues and primary human cells were approved by the IRBs of Academia Sinica, National Taiwan University Hospital, and National Cheng Kung University. Written informed consent was received from patients prior to their inclusion in the study. All mouse experiments were conducted with approval from the Experimental Animal Committee of Academia Sinica.

\section{Author contributions}

RHC conceived the project. YTW, JC, CWC, and RHC designed experiments. YTW, JC, CWC, CMC, and TYH conducted experiments and analyzed data. SYL analyzed TCGA data sets, RS provided technical help with the experimental metastasis model, and WWL provided human lung cancer specimens. JJ and YCW analyzed the human lung cancer specimens. SCY provided primary lung cancer cells, and KHC provided the KP mice. ICC and TSH provided the $W d r 4^{f l / f l}$ mice and WDR4 reagents. MZL provided conceptual and technical supports for tumor immunity studies. HCC provided instructions on lung cancer pathological analyses. YTW and RHC wrote the manuscript.

\section{Acknowledgments}

We thank Hsueh-Chi Sherry Yen (Institute of Molecular Biology, Academia Sinica, Taipei, Taiwan), Pan-Chyr Yang (National Taiwan University, Taipei, Taiwan), Szu-Hua Pan (National Taiwan University, Taipei, Taiwan), and Muh-Hwa Yang (National YangMing University, Taipei, Taiwan) for reagents; the National RNAi Core Facility for shRNAs; the Academia Sinica Animal Core Facility for IVIS analysis; the Institute of Biological Chemistry Histopathology Core Facility and the Institute of Biomedical Sciences (IBMS) Pathology Core Facility for tissue processing and pathology; the IBMS Flow Cytometry Core Facility for flow cytometry; Wan-Chen Hsieh Hsieh (Institute of Molecular Biology, Academia Sinica, Taipei, Taiwan) for instruction on flow cytometric analysis; and the Human Biobank, the Research Center of Clinical Medicine, and the Cancer Data Bank of National Cheng Kung University Hospital for patients' specimens. This work is supported by Ministry of Science and Technology (MOST) grant 104-2320-B-001-007 and National Health Research Institute grant EX-105-102-5NI.

Address correspondence to: Ruey-Hwa Chen, Institute of Biological Chemistry, Academia Sinica, Taipei 115, Taiwan. Phone: 886.2.27855696 ext.6020; Email:rhchen@gate.sinica.edu.tw.
1. Siegel R, Naishadham D, Jemal A. Cancer statistics, 2012. CA Cancer J Clin. 2012;62(1):10-29.

2. Hanahan D, Weinberg RA. Hallmarks of cancer: the next generation. Cell. 2011;144(5):646-674.

3. Hynes RO. The extracellular matrix: not just pretty fibrils. Science. 2009;326(5957):1216-1219.

4. Lu P, Weaver VM, Werb Z. The extracellular matrix: a dynamic niche in cancer progression. J Cell Biol. 2012;196(4):395-406.

5. Hanahan D, Coussens LM. Accessories to the crime: functions of cells recruited to the tumor microenvironment. Cancer Cell. 2012;21(3):309-322.
6. Shiao SL, Ganesan AP, Rugo HS, Coussens LM. Immune microenvironments in solid tumors: new targets for therapy. Genes Dev. 2011;25(24):2559-2572.

7. de Thé H, Chomienne C, Lanotte M, Degos L, Dejean A. The $\mathrm{t}(15 ; 17)$ translocation of acute promyelocytic leukaemia fuses the retinoic acid receptor alpha gene to a novel transcribed locus. Nature. 1990;347(6293):558-561.

8. Bernardi R, Pandolfi PP. Structure, dynamics and functions of promyelocytic leukaemia nuclear bodies. Nat Rev Mol Cell Biol. 2007;8(12):1006-1016.

9. Salomoni P, Ferguson BJ, Wyllie AH, Rich T. New insights into the role of PML in tumour suppression. Cell Res. 2008;18(6):622-640.

10. Reineke EL, Liu Y, Kao HY. Promyelocytic leukemia protein controls cell migration in response to hydrogen peroxide and insulin-like growth factor-1. J Biol Chem. 2010;285(13):9485-9492.

11. Sahin U, Lallemand-Breitenbach V, de Thé H. PML nuclear bodies: regulation, function and therapeutic perspectives. J Pathol. 2014;234(3):289-291.

12. Bernardi R, et al. PML inhibits HIF-1alpha translation and neoangiogenesis through repression of mTOR. Nature. 2006;442(7104):779-785. 
13. Carracedo A, et al. A metabolic prosurvival role for PML in breast cancer. J Clin Invest. 2012;122(9):3088-3100.

14. Wu HC, et al. USP11 regulates PML stability to control Notch-induced malignancy in brain tumours. Nat Commun. 2014;5:3214

15. Ito K, et al. PML targeting eradicates quiescent leukaemia-initiating cells. Nature. 2008;453(7198):1072-1078.

16. Gurrieri C, et al. Loss of the tumor suppressor PML in human cancers of multiple histologic origins. J Natl Cancer Inst. 2004;96(4):269-279.

17. Chen RH, Lee YR, Yuan WC. The role of PML ubiquitination in human malignancies. J Biomed Sci. 2012;19:81.

18. Yuan WC, et al. A Cullin3-KLHL2O Ubiquitin ligase-dependent pathway targets PML to potentiate HIF-1 signaling and prostate cancer progression. Cancer Cell. 2011;20(2):214-228.

19. Chen HY, et al. KLHL39 suppresses colon cancer metastasis by blocking KLHL20-mediated PML and DAPK ubiquitination. Oncogene. 2015;34(40):5141-5151.

20. Lin YC, et al. SCP phosphatases suppress renal cell carcinoma by stabilizing PML and inhibiting mTOR/HIF signaling. Cancer Res. 2014;74(23):6935-6946.

21. Rabellino A, et al. The SUMO E3-ligase PIAS1 regulates the tumor suppressor PML and its oncogenic counterpart PML-RARA. Cancer Res. 2012;72(9):2275-2284

22. Wolyniec $\mathrm{K}$, et al. E6AP ubiquitin ligase regulates PML-induced senescence in Myc-driven lymphomagenesis. Blood. 2012;120(4):822-832.

23. Angers S, Li T, Yi X, MacCoss MJ, Moon RT, Zheng N. Molecular architecture and assembly of the DDB1-CUL4A ubiquitin ligase machinery. Nature. 2006;443(7111):590-593.

24. He YJ, McCall CM, Hu J, Zeng Y, Xiong Y. DDB1 functions as a linker to recruit receptor WD40 proteins to CUL4-ROC1 ubiquitin ligases. Genes Dev. 2006;20(21):2949-2954.

25. Higa LA, Wu M, Ye T, Kobayashi R, Sun H, Zhang H. CUL4-DDB1 ubiquitin ligase interacts with multiple WD40-repeat proteins and regulates histone methylation. Nat Cell Biol. 2006;8(11):1277-1283.

26. Jin J, Arias EE, Chen J, Harper JW, Walter JC. A family of diverse Cul4-Ddb1-interacting proteins includes Cdt2, which is required for S phase destruction of the replication factor Cdt1. Mol Cell. 2006;23(5):709-721.

27. Shaheen R, et al. Mutation in WDR4 impairs tRNA m(7)G46 methylation and causes a distinct form of microcephalic primordial dwarfism. Genome Biol. 2015;16:210.

28. Xu C, Min J. Structure and function of WD40 domain proteins. Protein Cell. 2011;2(3):202-214

29. Beavis PA, Stagg J, Darcy PK, Smyth MJ. CD73: a potent suppressor of antitumor immune respons- es. Trends Immunol. 2012;33(5):231-237.

30. Stagg J, et al. Anti-CD73 antibody therapy inhibits breast tumor growth and metastasis. Proc Nat Acad Sci USA . 2010;107(4):1547-1552.

31. Smith HW, Marshall CJ. Regulation of cell signalling by uPAR. Nat Rev Mol Cell Biol. 2010;11(1):23-36

32. Malle E, Sodin-Semrl S, Kovacevic A. Serum amyloid A: an acute-phase protein involved in tumour pathogenesis. Cell Mol Life Sci. 2009;66(1):9-26.

33. Krishnamachary B, et al. Regulation of colon carcinoma cell invasion by hypoxia-inducible factor 1. Cancer Res. 2003;63(5):1138-1143.

34. Synnestvedt K, et al. Ecto-5'-nucleotidase (CD73) regulation by hypoxia-inducible factor- 1 mediates permeability changes in intestinal epithelia. JClin Invest. 2002;110(7):993-1002.

35. Laufs S, Schumacher J, Allgayer H. Urokinasereceptor (u-PAR): an essential player in multiple games of cancer: a review on its role in tumor progression, invasion, metastasis, proliferation/ dormancy, clinical outcome and minimal residual disease. Cell Cycle. 2006;5(16):1760-1771.

36. Carmeliet $\mathrm{P}$, et al. Urokinase-generated plasmin activates matrix metalloproteinases during aneurysm formation. Nat Genet. 1997;17(4):439-444.

37. Condemine W, et al. Characterization of endogenous human promyelocytic leukemia isoforms. Cancer Res. 2006;66(12):6192-6198.

38. O'Reilly MS, et al. Angiostatin: a novel angiogenesis inhibitor that mediates the suppression of metastases by a Lewis lung carcinoma. Cell. 1994;79(2):315-328.

39. Cheng IC, Chen BC, Shuai HH, Chien FC, Chen P, Hsieh TS. Wuho is a new member in maintaining genome stability through its interaction with Flap endonuclease 1. PLoS Biol. 2016;14(1):e1002349.

40. DuPage M, Dooley AL, Jacks T. Conditional mouse lung cancer models using adenoviral or lentiviral delivery of Cre recombinase. Nat Protoc. 2009;4(7):1064-1072.

41. Csóka B, et al. Adenosine promotes alternative macrophage activation via $\mathrm{A} 2 \mathrm{~A}$ and $\mathrm{A} 2 \mathrm{~B}$ receptors. FASEB J. 2012;26(1):376-386.

42. Rao S, et al. A dual role for autophagy in a murine model of lung cancer. Nat Commun. 2014;5:3056.

43. Li X, Kostareli E, Suffner J, Garbi N, Hämmerling GJ. Efficient Treg depletion induces T-cell infiltration and rejection of large tumors. Eur J Immunol. 2010;40(12):3325-3335.

44. Teng MW, Ngiow SF, von Scheidt B, McLaughlin N, Sparwasser T, Smyth MJ. Conditional regulatory $\mathrm{T}$-cell depletion releases adaptive immunity preventing carcinogenesis and suppressing established tumor growth. Cancer Res. 2010;70(20):7800-7809.

45. Emanuele MJ, et al. Global identification of modular cullin-RING ligase substrates. Cell.
2011;147(2):459-474.

46. Beroukhim R, et al. The landscape of somatic copy-number alteration across human cancers. Nature. 2010;463(7283):899-905.

47. Kessenbrock K, Plaks V, Werb Z. Matrix metalloproteinases: regulators of the tumor microenvironment. Cell. 2010;141(1):52-67.

48. Mazar AP. Urokinase plasminogen activator receptor choreographs multiple ligand interactions: implications for tumor progression and therapy. Clin Cancer Res. 2008;14(18):5649-5655.

49. Kuo HY, et al. PML represses lung cancer metastasis by suppressing the nuclear EGFR-mediated transcriptional activation of MMP2. Cell Cycle. 2014;13(19):3132-3142.

50. El Asmi F, Maroui MA, Dutrieux J, Blondel D, Nisole S, Chelbi-Alix MK. Implication of PMLIV in both intrinsic and innate immunity. PLOS Pathog. 2014;10(2):e1003975.

51. Spranger S, Bao R, Gajewski TF. Melanomaintrinsic $\beta$-catenin signalling prevents antitumour immunity. Nature. 2015;523(7559):231-235.

52. Ihara $S$, et al. Inhibitory roles of signal transducer and activator of transcription 3 in antitumor immunity during carcinogen-induced lung tumorigenesis. Cancer Res. 2012;72(12):2990-2999.

53. Wang T, et al. Regulation of the innate and adaptive immune responses by Stat-3 signaling in tumor cells. Nat Med. 2004;10(1):48-54.

54. Peng D, et al. Epigenetic silencing of TH1-type chemokines shapes tumour immunity and immunotherapy. Nature. 2015;527(7577):249-253.

55. Iannello A, Thompson TW, Ardolino M, Lowe SW, Raulet DH. p53-dependent chemokine production by senescent tumor cells supports NKG2D-dependent tumor elimination by natural killer cells. J Exp Med. 2013;210(10):2057-2069.

56. Dunn GP, Bruce AT, Ikeda H, Old LJ, Schreiber RD. Cancer immunoediting: from immunosurveillance to tumor escape. Nat Immunol. 2002;3(11):991-998.

57. Zou W. Immunosuppressive networks in the tumour environment and their therapeutic relevance. Nat Rev Cancer. 2005;5(4):263-274.

58. Cui TX, et al. Myeloid-derived suppressor cells enhance stemness of cancer cells by inducing microRNA101 and suppressing the corepressor CtBP2. Immunity. 2013;39(3):611-621.

59. Kryczek I, et al. IL-22(+)CD4(+) T cells promote colorectal cancer stemness via STAT3 transcription factor activation and induction of the methyltransferase DOT1L. Immunity. 2014;40(5):772-784.

60. Yeh CT, et al. Trifluoperazine, an antipsychotic agent, inhibits cancer stem cell growth and overcomes drug resistance of lung cancer. Am J Respir Crit Care Med. 2012;186(11):1180-1188.

61. Wang WJ, et al. The tumor suppressor DAPK is reciprocally regulated by tyrosine kinase Src and phosphatase LAR. Mol Cell. 2007;27(5):701-716. 